\title{
Globular cluster content and evolutionary history of NGC 147
}

\author{
M. Sharina ${ }^{1,2}$ and E. Davoust ${ }^{2}$ \\ 1 Special Astrophysical Observatory, Russian Academy of Sciences, N. Arkhyz, KChR 369167, Russia \\ e-mail: sme@sao.ru \\ ${ }^{2}$ Laboratoire d'Astrophysique de Toulouse-Tarbes, Université de Toulouse, CNRS, 14 avenue E. Belin, 31400 Toulouse, France
}

Received 7 November 2008 / Accepted 28 January 2009

\begin{abstract}
Context. Globular clusters are representative of the oldest stellar populations. It is thus essential to have a complete census of these systems in dwarf galaxies, from which more massive galaxies are progressively formed in the hierarchical scenario.

Aims. We present the results of spectroscopic observations of eight globular cluster candidates in NGC 147, a satellite dwarf elliptical galaxy of M 31. Our goal is to make a complete inventory of the globular cluster system of this galaxy, determine the properties of their stellar populations, and compare these properties with those of systems of globular clusters in other dwarf galaxies.

Methods. The candidates were identified on Canada-France-Hawaii telescope photographic plates. Medium resolution spectra were obtained with the SCORPIO spectrograph at the prime focus of the $6 \mathrm{~m}$ telescope of the Russian Academy of Sciences. They were analyzed using predictions of stellar population synthesis models.

Results. We were able to confirm the nature of all eight candidates, three of which (GC5, GC7, and GC10) are indeed globular clusters, and to estimate evolutionary parameters for the two brightest ones and for Hodge II. The bright clusters GC5 and GC7 appear to have metallicities $([\mathrm{Z} / \mathrm{H}] \sim-1.5 \div-1.8)$ that are lower than the oldest stars in the galaxy. The fainter GC Hodge II has a metallicity $[\mathrm{Z} / \mathrm{H}]=$ -1.1 , similar to that of the oldest stars in the galaxy. The clusters GC5 and GC7 have low alpha-element abundance ratios. The mean age of the globular clusters in NGC 147 is $9 \pm 1$ Gyr. We also measured the radial velocities of Hodge II and IV, and derived a mass of NGC 147 in good agreement with the value from the literature. The frequency, $S_{n}=6.4$, and mass fraction, $T=14$ of globular clusters in NGC 147 appear to be higher than those for NGC 185 and 205.

Conclusions. Our results indicate that the bright clusters GC5, GC7, and Hodge III formed in the main star-forming period 8-10 Gyr ago, while the fainter clusters Hodge I and II formed together with the second generation of field stars.
\end{abstract}

Key words. galaxies: dwarf - galaxies: star clusters - galaxies: abundances

\section{Introduction}

The relative frequency of dwarf ellipticals (dEs) and Sculptortype spheroidal galaxies (dSph) depends on the environment, and one can expect that the properties of their populations of globular clusters (GCs) also do. In the Local Volume (LV), which spans a region of up to $\sim 10 \mathrm{Mpc}$ from the Sun, the most frequent types are low surface-brightness dwarf irregular and spheroidal galaxies. In contrast, there are hundreds of dEs in the nearest rich clusters of galaxies Virgo and Fornax (Binggeli et al. 1985; Ferguson 1989).

NGC 147, 185, 205, and M 32 are the brightest early-type dwarf galaxies in the LV (Mateo 1998; van den Bergh 2000; Karachentsev et al. 2004). Their absolute $V$-magnitudes range from -15.1 for NGC 147 to -16.7 for M 32, making them more luminous than the Sculptor-type spheroidal galaxies (dSph), whose absolute $V$-magnitudes are between -8 and -13 . They also have higher central and mean surface brightnesses (see e.g. Karachentseva et al. 1985). Their colors resemble those of old, gas-free stellar systems, such as the Galactic globular clusters.

The four satellite galaxies of M 31 were first resolved into individual stars by Baade (1944a,b). Their distances are well determined, and the properties of their stellar populations have been thoroughly studied (e.g. Mould et al. 1983; Davidge 1994, 2005; Lee et al. 1993; Grebel 2000; McConnachie 2005;
Butler \& Martínez-Delgado 2005; Dolphin 2005). NGC 147 is composed of old (90\%) and intermediate-age stellar populations (Mould et al. 1983; Davidge 2005). The percentage of intermediate-age stellar populations is higher and the metallicities and metallicity spreads are greater in the other dEs.

NGC 185, 205, and M32 have prominent peculiarities in their structure and stellar content. It has been argued that the gas-free M 32 is a stripped spiral (Bekki et al. 2001; Graham 2002). NGC 185 and 205 contain neutral hydrogen and molecular gas, dust, and young stars (Baade 1944a,b; Hodge 1963; Lee et al. 1993; Butler \& Martínez-Delgado 2005; Young \& Lo 1997; Sage et al. 1998). Both galaxies reveal bright nuclei with a young star-forming region in the first one, and a relatively young star cluster in the other (see e.g. Da Costa \& Mould 1988; Young \& Lo 1997; Sharina et al. 2006b).

NGC 185 and 147 have similar photometric and structural properties (Mateo 1998). van den Bergh (1998) has shown that these galaxies form a binary system. However, there are no indications of a tidal bridge between them (Battinelli \& Demers 2004). They are slightly nearer to our Galaxy than to M 31, and are not supposed to have had close passages to M 31. The differences in the gas content and properties of stellar populations between NGC 185 and 147 are not well understood. The observed gas content in NGC 185 and 205 is explained by stellar mass return (Welch et al. 1998). In contrast, in NGC 147 and 
M 32 the mass of the interstellar medium is much lower than the one expected from material ejected from evolved stars (Sage et al. 1998).

The most prominent properties of stellar populations, common to NGC 147, 185, and 205, are i) sizable globular cluster systems, where the majority of GCs are old and metalpoor (Da Costa \& Mould 1988; van den Bergh 2000; Sharina et al. 2006b); ii) the presence of intermediate-age stars (Davidge 2005; Saha et al. 1990), planetary nebulae (Richer \& McCall 1995; Corradi et al. 2005; Gonçalves et al. 2006); and iii) similar median metallicities of old stars, $[\mathrm{Fe} / \mathrm{H}] \sim-0.9$ dex (Butler \& Martínez-Delgado 2005; Davidge 2005). It is noteworthy that the stellar halo of M 31 at galactocentric distances $R \geq 60 \mathrm{kpc}$ is more metal-poor $\langle[\mathrm{Fe} / \mathrm{H}]\rangle=-1.26 \pm 0.10 \operatorname{dex}(\sigma=0.72)$ (Kalirai et al. 2006).

Recent observational results have demonstrated that the formation of GCs occurs under high densities and pressures during the most powerful star forming events in the life of a galaxy. This is why the determination of evolutionary parameters of GCs is important for understanding the star formation histories of individual galaxies and the formation of cosmological structures in general. Four globular clusters were found in NGC 147 by Baade (1944b), who indicated the existence of two GCs and a "semistellar nucleus", and by Hodge (1976). Accurate positions for Hodge I-III were given by Ford et al. (1977). Da Costa $\&$ Mould (1988) were the first to obtain spectra for the brightest GCs in NGC 147, 185, and 205, and estimated their mean abundance to be considerably less than that of the corresponding field halo stars. They concluded that the GCs were formed at the earliest epochs of galactic formation along with the first stars. Sharina et al. (2006b, hereafter SAP06) obtained high $S / N$ ratio, medium-resolution spectra for two GCs in NGC 147, 6 GCs in NGC 185, and 5 GCs in NGC 205, and estimated evolutionary parameters for them and for diffuse galactic light regions near the centers of NGC 205 and NGC 185.

Our paper continues the aforementioned spectroscopic studies of GCs in NGC 147. We found three additional globular clusters in NGC 147, obtained their spectra on the $6 \mathrm{~m}$ telescope, and estimated ages, metallicities and abundance ratios for the two brightest, GC5 and GC7, and for Hodge II. Additionally, we obtained radial velocities for Hodge IV and GC10. These new data, together with others gathered from the literature, enabled us to propose a scenario for the evolutionary history of NGC 147. Our paper is organised as follows. In Sect. 2 we describe our observations and the data reduction. In Sect. 3 we explain our methods of age and metallicity determination. We discuss the obtained results in Sect. 4, and formulate our conclusions in Sect. 5. In the Appendix we give a mass estimate for NGC 147 using radial velocities for the GCs obtained by us and by SAP06, and present absorption-line indices measured in the spectra of GC5, GC7, and Hodge II, as well as comparisons of our Lick index measurements with SSP model predictions.

\section{Observations and data reduction}

\subsection{Images}

This project started when non-stellar objects were discovered on photographic plates of NGC 147 obtained at the Cassegrain focus of the $3.6 \mathrm{~m}$ Canada-France-Hawaii telescope (CFHT). These plates were in fact the first ones ever taken at this focus of the telescope. Follow-up CCD imaging of the globular
Table 1. Images used for GC searches, astrometry, and photometry.

\begin{tabular}{lclc}
\hline \hline Object/Filter & \multicolumn{1}{c}{ Date } & \multicolumn{1}{c}{ Exposure $(\mathrm{s})$} & Seeing \\
\hline CFHT 3.6 m telescope, photographic plates \\
NGC 147 $(B)$ & $16 / 08 / 83$ & 1800 & 1.0 \\
NGC 147 $(V)$ & $16 / 08 / 83$ & 1800 & 1.1 \\
Pic-du-Midi 2 & m telescope, CCD & \\
Ho III $(V, R)$ & $02 / 11 / 87$ & 1800 & 1.4 \\
5+6 $(B, V, R)$ & $16 / 12 / 87$ & 1200 & 1.0 \\
GC7 $(R)$ & $15 / 12 / 87$ & $720 \times 2$ & 1.2 \\
GC7 $(R)$ & $16 / 12 / 87$ & 1800 & 1.2 \\
GCC $8(V)$ & $05 / 08 / 88$ & $1800 \times 2$ & 1.1 \\
GCC9 $(V)$ & $05 / 08 / 88$ & 1800 & 1.1 \\
GCC11 $(V)$ & $07 / 08 / 88$ & 1800 & 1.3 \\
GCC11 $(R)$ & $08 / 08 / 88$ & 1800 & 0.9 \\
GCC12 $(V)$ & $07 / 08 / 88$ & 1200 & 1.1 \\
6 m telescope of Russian A.S., CCD & \\
GC10 $(R)$ & $14 / 08 / 07$ & $30 \times 2$ & 1.2 \\
GC10 $(V)$ & $03 / 08 / 08$ & $30 \times 2$ & 1.0 \\
GCC12 $(R)$ & $14 / 08 / 07$ & $30 \times 2$ & 1.2 \\
\hline \multicolumn{5}{c}{}
\end{tabular}

cluster candidates (GCCs) were obtained under good seeing conditions at the F/10 Cassegrain focus of the $2 \mathrm{~m}$ Bernard-Lyot telescope of Pic-du-Midi Observatory. These preliminary imaging observations are summarised in Table 1, together with those obtained during the spectroscopic runs 2007, 2008. The exact positions of the GCCs were determined by Le Campion at Bordeaux Observatory: a photographic plate of the field was obtained at the astrographic telescope, the positions of 64 stars in common with the CFHT plates were measured on all plates, as well as those of the GCCs, and their precise coordinates determined using 47 fundamental stars. Finally, the coordinates of the GCCs were derived with a precision of $0.55^{\prime \prime}$. They are given in Table 2, together with their magnitudes and colors measured on the CCD images. The positions of the GCCs and of the four known globular clusters (Hodge I to IV) are shown in Fig. 1, which is a reproduction of the CFHT photographic plate in the $B$-band. CCD images of the spectroscopically confirmed GCCs are presented in Fig. 2.

$V$ and $R$ magnitudes in the Johnson-Cousins photometric system were obtained for the GCCs using the pre-imaging observations, with typical errors $\sim 0.3 \mathrm{mag}$. The main contributors to the errors are uncertainties of transformations between the instrumental and standard systems using secondary standard stars and random photometric errors significant at low exposure times and with old matrices. We could not use photometric standards to calibrate our measurements because atmospheric conditions were unstable during the observations. The magnitudes are gathered in Table 2 together with heliocentric radial velocities and projected distances from the center of the galaxy. Assuming a distance modulus $(m-M)_{0}=23.95 \mathrm{mag}$ (McConnachie et al. 2004) and a Galactic extinction $A_{V}=0.58$ (Schlegel et al. 1998), the absolute $V$ magnitudes of GC5, GC7, GC10, and Hodge IV are $-5.7,-7.1,-4.0$, and -4.7 , respectively. The absolute magnitudes of Hodge I-III were determined by Sharina \& Puzia (2009) on HST WFPC2 images. The resulting mean absolute magnitude of GCs in NGC 147 is $M_{V} \sim-6.0 \mathrm{mag}$. This value is fainter than the one for globular cluster systems in giant galaxies, which is $M_{V}=-7.5$, fairly independent of the parent galaxy morphological type (Harris 1991).

Full widths at half maxima (FWHM) for GC5, GC7, and GC10 approximately correspond to half-light radii of $2,1.5$, and 3 pc, respectively, if one convolves artificial two-dimensional 


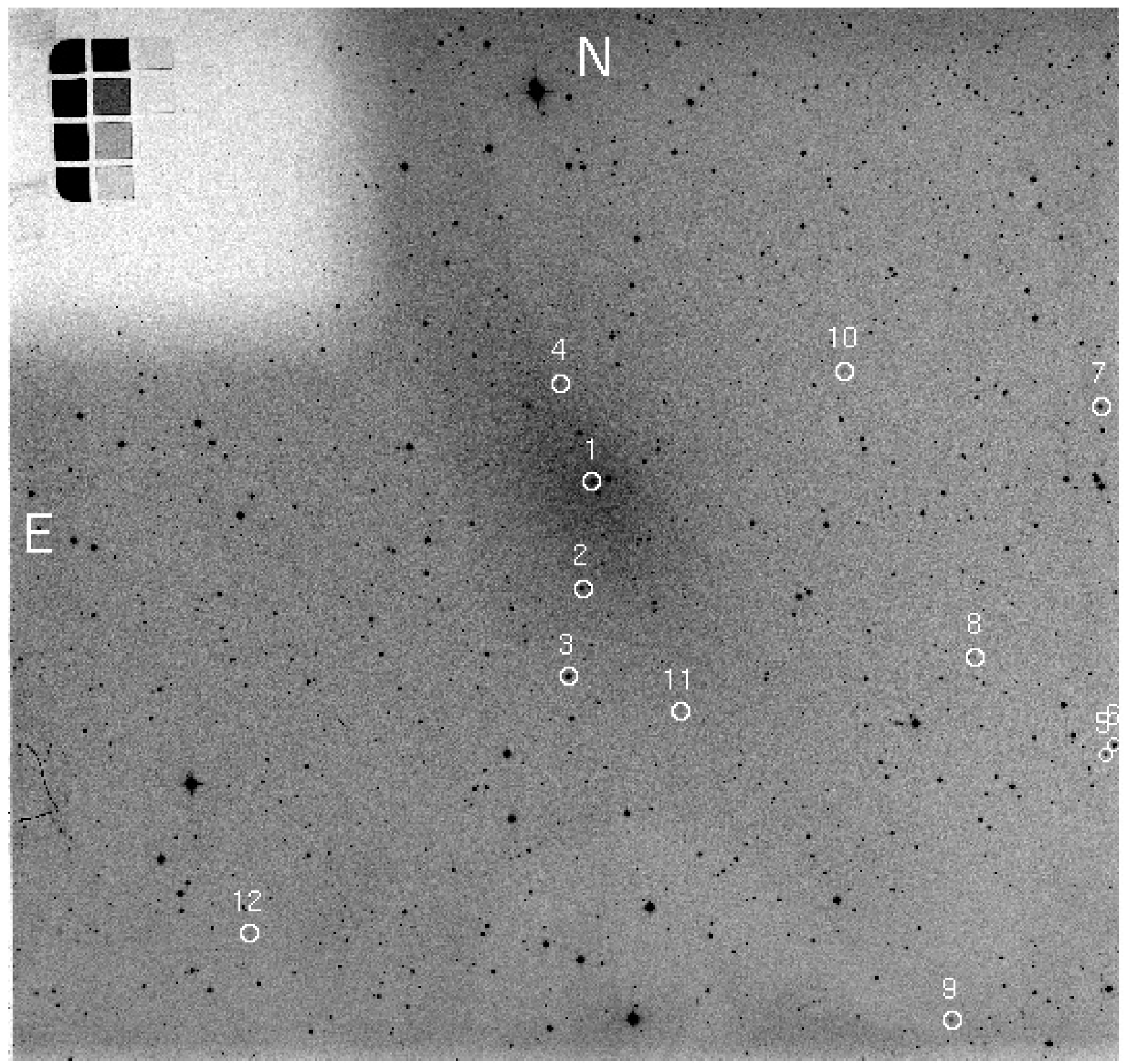

Fig. 1. Reproduction of the CFHT $B$-band photographic plate. The field of view is $18^{\prime}$. The globular clusters and candidates are indicated.

frames calculated using the Gaussian functions of corresponding FWHM with the observed stellar point-spread functions. These values are typical of GCs in dwarf ellipticals in the Virgo cluster (Jordán et al. 2005).

\subsection{Spectra}

The long-slit spectroscopic observations were performed with the SCORPIO spectrograph (Afanasiev \& Moiseev 2005), installed at the prime focus of the $6 \mathrm{~m}$ telescope of the Russian Academy of Sciences. The journal of the spectroscopic observations is given in Table 3. We used the CCD detector EEV42-40, the grism VPHG1200g (1200 lines/mm) with a spectral resolution $\sim 5 \AA$, the spectral range 3800-5400 $\AA$. The slit width was $6^{\prime} \times 1^{\prime \prime}$. We observed Lick standard stars from the list of Worthey et al. (1994) for calibration of our instrumental absorption-line system into the Lick standard one (Worthey 1994; Worthey \& Ottaviani 1997).

Although the weather was mostly good during the observing run of 2007, thin cirrus developed from time to time, and the end of the night 13/08/07 was lost due to a sudden thunderstorm, preventing us from observing all the standard stars. Some exposures were lost in the night 14/08/07 due to disrepair in the filter wheel. The CCD detector became fogged over in the central part $(\sim 700 \times 700$ pixels $)$ in the final night 2008 , forcing us to use only part of the detector.

The data reduction and analysis were performed using the European Southern Observatory Munich Image Data Analysis System (MIDAS) (Banse et al. 1983), and the Image Reduction 

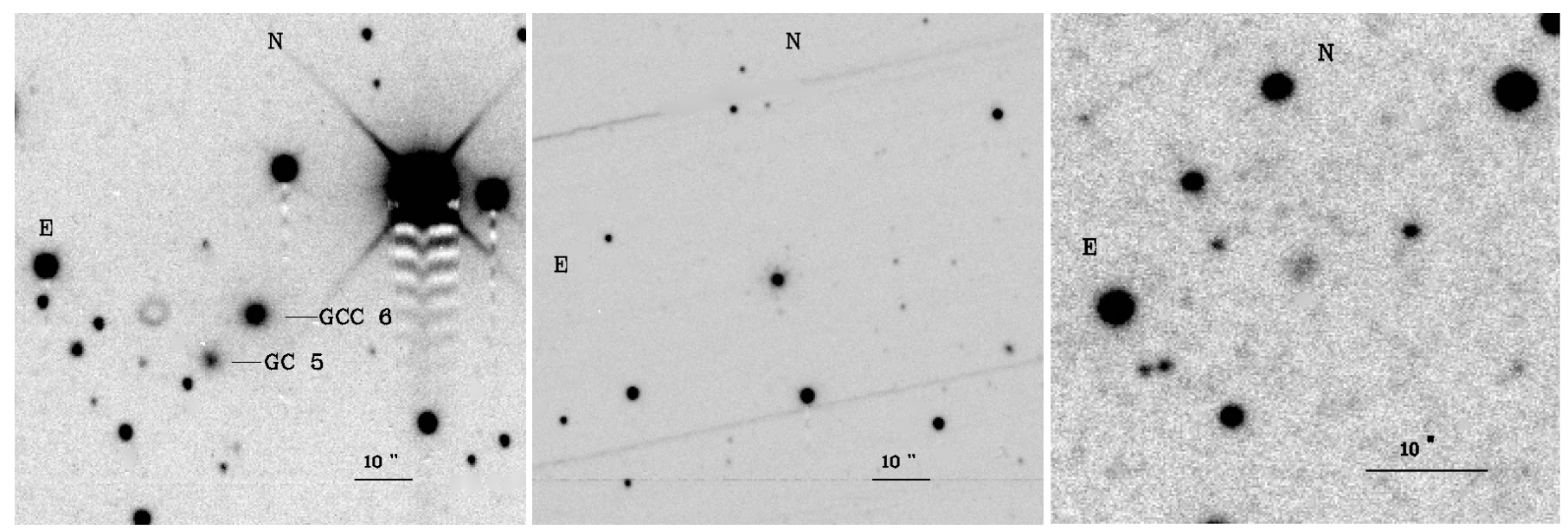

Fig. 2. Pic-du-Midi and $6 \mathrm{~m}$ telescope images of GC5, GC7, and GC10, taken in good atmospheric conditions (seeing $\sim 1^{\prime \prime}$ ).

Table 2. Coordinates, magnitudes, heliocentric radial velocities and projected distances of GCs and GCCs. Indices are 1: from Sharina \& Puzia (2009); 2: $(V-I)$ instead of $(V-R)$; 3: from SAP06.

\begin{tabular}{|c|c|c|c|c|c|}
\hline Object & RA (2000) Dec & $V$ & $V-R$ & $\begin{array}{c}V_{\mathrm{h}}, \\
\mathrm{km} \mathrm{s}^{-1}\end{array}$ & $\begin{array}{l}d_{\mathrm{pr}}, \\
\mathrm{kpc}\end{array}$ \\
\hline GC5 & $003223.4+482544.7$ & 18.8 & 0.8 & $\begin{array}{l}-187 \\
\pm 15\end{array}$ & 2.1 \\
\hline GC7 & $003222.2+483127.5$ & 17.4 & 0.8 & $\begin{array}{c}-198 \\
\pm 10\end{array}$ & 1.8 \\
\hline GC10 & $003248.6+483212.4$ & 20.5 & 0.8 & $\begin{array}{c}-180 \\
\pm 30\end{array}$ & 0.9 \\
\hline H I & $003312.2+483032.6$ & $18.5^{1}$ & $1.4^{1,2}$ & $\begin{array}{c}-107^{3} \\
\pm 30\end{array}$ & $1 \mathrm{E}-3$ \\
\hline H II & $003313.6+482848.3$ & $18.7^{1}$ & $1.2^{1,2}$ & $\begin{array}{c}-189 \\
\pm 25\end{array}$ & 0.7 \\
\hline H III & $003315.1+482723.4$ & $16.8^{1}$ & $1.1^{1,2}$ & $\begin{array}{c}-118^{3} \\
\pm 30\end{array}$ & 0.4 \\
\hline H IV & $003315.0+483209.7$ & 19.8 & 0.9 & $\begin{array}{l}-235 \\
\pm 35\end{array}$ & 0.4 \\
\hline GCC6 & $003222.9+482548.9$ & 17.4 & 1.0 & 19200 & \\
\hline GCC8 & $003235.3+482728.9$ & 19.4 & - & 27000 & \\
\hline GCC9 & $003239.0+482136.0$ & 18.5 & - & 25800 & \\
\hline GCC11 & $003304.3+482646.1$ & 19.9 & 0.5 & 48000 & \\
\hline GCC12 & $003347.2+482324.4$ & 19.8 & 0.9 & 3000 & \\
\hline
\end{tabular}

and Analysis Facility (IRAF) software system ${ }^{1}$. Cosmic-ray removal was done with the FILTER/COSMIC program in MIDAS. The standard procedure of primary reduction was applied to each two-dimensional spectrum. Wavelength calibration was carried out using an arc spectrum taken at the same pointing positions as the cluster spectrum before or after the object exposure. The dispersion solution provides the accuracy of the wavelength calibration $\sim 0.08 \AA$. A typical dispersion was $0.88 \AA /$ pix. The wavelength zeropoint shifted during the night by up to 2 pixels. It was checked using the [OI] $\lambda 5577$ night sky line in the dispersioncorrected spectra. Extraction of the spectra (Horne 1986) was made using the IRAF procedure apsum.

1 http://iraf.noao.edu/
Table 3. Journal of spectroscopic observations.

\begin{tabular}{lclc}
\hline \hline Object & Date & Exposure (s) & Seeing \\
\hline GC7 & $11 / 08 / 07$ & $4 \times 900$ & 2.0 \\
Hodge II+GCC11 & $11 / 08 / 07$ & 900 & 2.0 \\
GCC9 & $11 / 08 / 07$ & $3 \times 900$ & 2.0 \\
GC5+GCC8 & $13 / 08 / 07$ & 1200 & 2.0 \\
Hodge II+Hodge 4 & $13 / 08 / 07$ & $2 \times 900$ & 1.2 \\
GC10 & $14 / 08 / 07$ & 900 & 2.0 \\
GCC12 & $14 / 08 / 07$ & $3 \times 900$ & 2.0 \\
GCC6 & $03 / 08 / 08$ & $3 \times 600$ & 1.7 \\
GC10 & $03 / 08 / 08$ & $3 \times 900$ & 2.3 \\
HD 7010 & $11,14 / 08 / 07$ & 20 & 2.0 \\
HD 7010 & $03 / 08 / 08$ & 10 & 2.3 \\
HD 2665 & $11,14 / 08 / 07$ & 10 & 2.0 \\
HD 2665 & $03 / 08 / 08$ & 5 & 2.3 \\
HR 1805 & $11 / 08 / 07$ & 2 & 2.0 \\
GRW+70 5824 & $11 / 08 / 07$ & 100 & 2.0 \\
HZ4 & $13 / 08 / 07$ & 150 & 2.0 \\
\hline
\end{tabular}

After wavelength calibration and sky subtraction, the spectra were corrected for extinction and flux-calibrated using the observed spectrophotometric standard stars GRW $+70^{\circ} 5824$, and HZ4 (Oke 1990). Due to non-photometric conditions, the flux calibration is approximate. Finally, all one-dimensional spectra of each object were summed to increase the $S / N$ ratio.

The radial velocities of the objects were determined using the method of Tonry \& Davis (1979). We used several radial velocity standards, the PEGASE.HR synthetic spectra ${ }^{2}$ for crosscorrelation. To estimate radial velocities of remote emission-line galaxies, we used the template starburst galaxy spectral energy distributions SB6 shifted to the redshift of the studied objects. The derived heliocentric radial velocities are listed in Table 2. The resulting normalized spectra of GCs and GCCs, together with spectra of standard stars, and cross-correlation functions are shown in Fig. 3.

${ }^{2}$ http://www2.iap.fr/pegase/pegasehr 

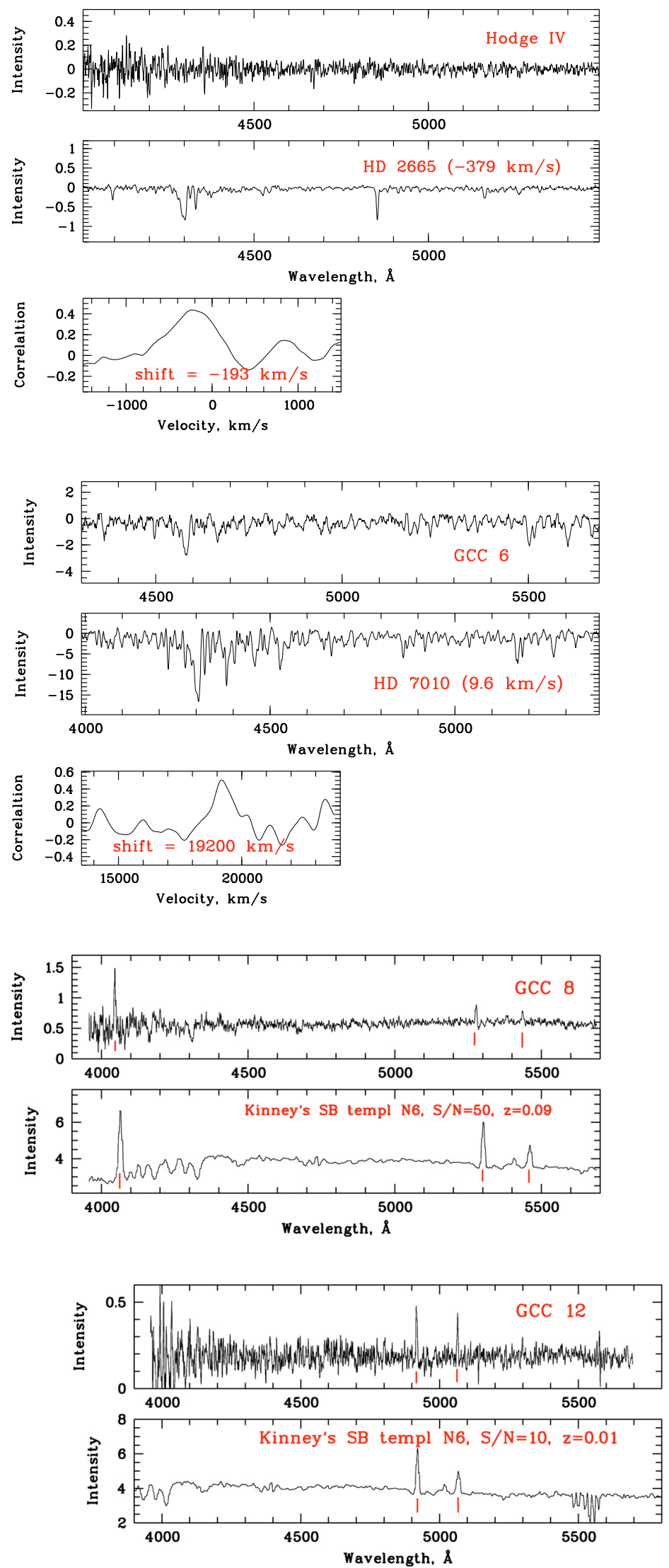

Fig. 3. Spectra and cross-correlation functions of globular clusters Hodge IV and GC10, and of GCCs classified as remote galaxies. Bright emission-line features in the spectra of distant starburst galaxies ([OII] 3727, $\mathrm{H} \beta$, [OIII] 4959, 5007) are indicated. In these cases templates of Kinney et al. (1996) are shown. They were shifted according to the redshifts.
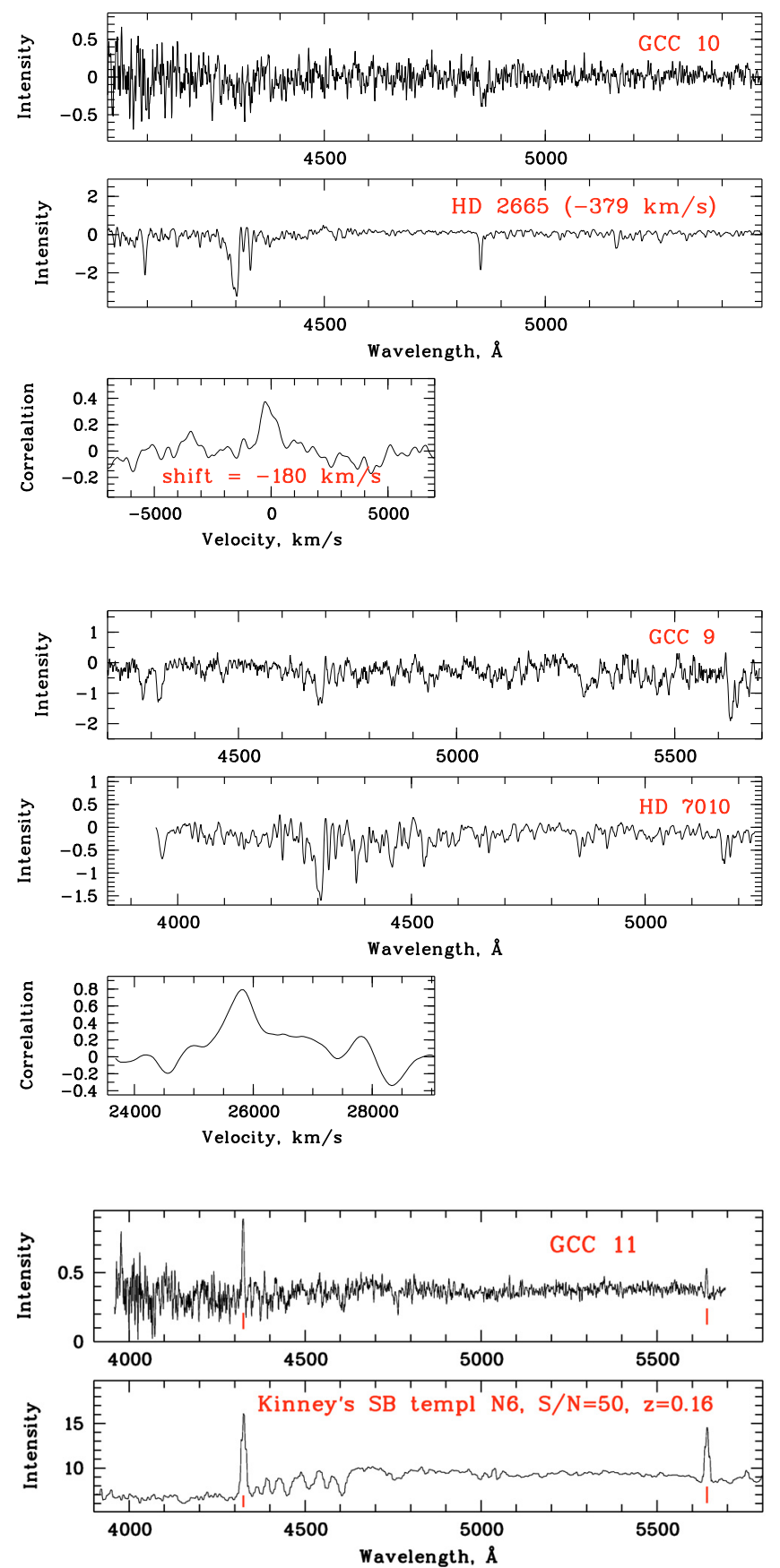

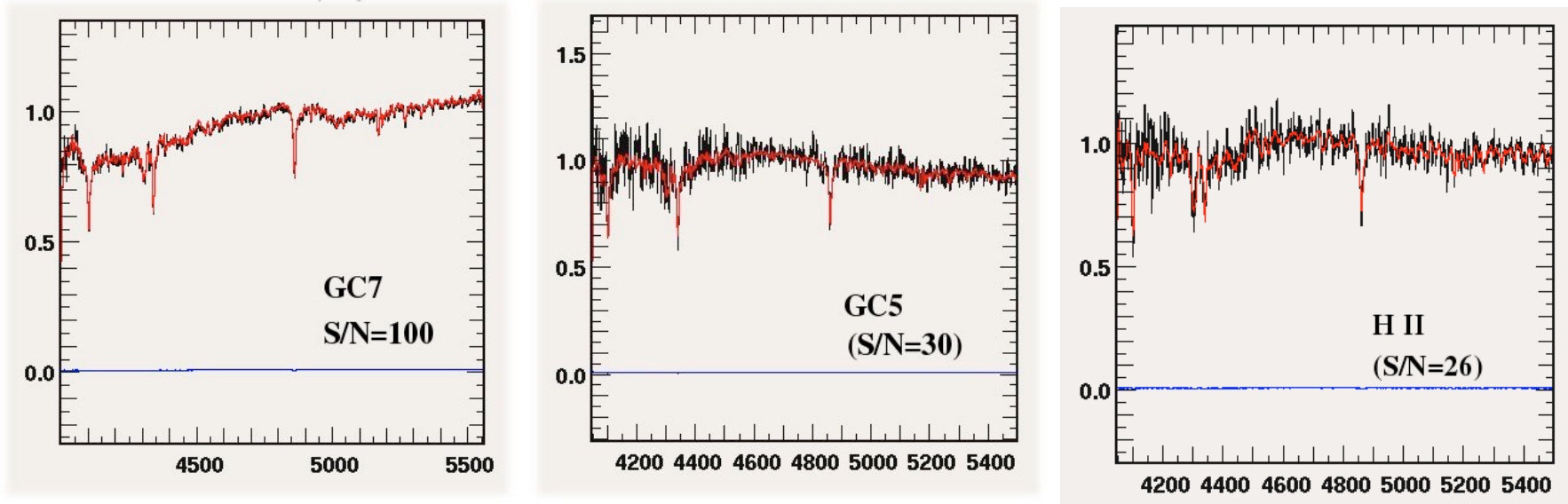

Fig. 4. Spectra of GC7, GC5, and Hodge II scaled to the original continuum at $\lambda=5000 \AA$ A. PEGASE.HR model spectra fitted by the STECKMAP program (Ocvirk et al. 2006a,b) are overplotted.

The flux-calibrated spectra of the two brightest spectroscopically confirmed GCs and of Hodge II scaled to their continuum at $\lambda=5000 \AA$ are shown in Fig. 4 .

\section{Results}

\subsection{Methods of age, metallicity and $\alpha$-element abundance ratio determination}

In the analysis of the medium-resolution spectra, we applied three methods for determining the evolutionary parameters. The first one is our main working method (see also SAP06) for comparing intensities of absorption-line features in spectra of GCs using the Lick-index definitions of Worthey (1994) and Worthey \& Ottaviani (1997) with predictions of SSP models (Thomas et al. 2003, 2004). Two additional approaches proposing full spectrum fitting using the PEGASE.HR model spectra (Le Borgne et al. 2004) helped us understand how the choice of models, the number of spectral features involved in the analysis, imperfection of the flux calibration, and uncertainties of the Lick index zeropoints influence the derived parameters. Anticipating on the results, we point out that the three methods mentioned above gave similar ages and metallicities within the errors for the $\mathrm{GCs}^{3}$. In the following we describe the methods and results in detail.

\subsubsection{Lick indices}

The most commonly used method for disentangling agemetallicity degeneracy effects is that of the Lick indices (e.g. Worthey et al. 1994; Worthey 1994; Worthey \& Ottaviani 1997; Faber 1973; Proctor et al. 2004; Puzia et al. 2005b). The quality of the spectra allowed us to accurately measure the absorptionline indices and estimate ages, metallicities, and $\alpha$-element abundance ratios $([\alpha / \mathrm{Fe}])$ of our GCs by comparison of the measured values with the model ones.

\footnotetext{
${ }^{3}$ We use the standard definition, $[X / Y]=\log (X / Y)-\log \left(X_{\odot} / Y_{\odot}\right)$, where $X$ and $Y$ are masses of specific elements. $[\mathrm{Z} / \mathrm{H}]$ is the overall metallicity.
}

We used a number of Lick standard stars observed with the long-slit unit at the SCORPIO spectrograph from previous programs to calculate zeropoints of transformation of our Lick index measurements into the standard system (Worthey 1994) (see Table 4). The range of index values covered by our standard stars is given in Col. 4 of Table 4 .

The calculated zeropoint transformations to the Lick/IDS system agree with those given in Sharina et al. (2006a). However, we point out that we managed to obtain spectra for only 3 Lick standard stars during the observing runs of 2007,2008 . The rms errors of zeropoints are consequently large. So, for better confidence in the results we used here additional methods of evolutionary parameter determination, independent of the Lick index one, (see Sects. 3.1.2, 3.1.3 for details).

Absorption-line indices for the spectroscopically confirmed GCs with $S / N \geq 25$, measured by the GONZO program (Puzia et al. 2002) and transformed into the Lick system are listed in Tables C.1, C.2. The spectra were degraded to the resolution of the Lick system before measuring the indices.

To estimate the evolutionary parameters we use a three-dimensional interpolation and $\chi^{2}$ approximation routine (SAP06), and SSP models of Thomas et al. (2003, 2004). The $\chi^{2}$ minimization technique has the advantage of using all available indices to determine the evolutionary parameters (Proctor et al. 2004). This reduces the influence of individual index uncertainties on the results. Systematic errors arise because of variable atmospheric transparency, instrumental problems, and imperfections of data reduction: uncertainties of flux calibration and sky subtraction, scattered light effects, spectral wavelength calibration and radial velocity determination, corrections from spectral resolution, seeing, focus and velocity dispersion, response deviations from linearity, the contribution from nebular absorption lines. Uncertainties of transformation into the Lick system appear to be significant when the number of observed standard stars is small. Statistical index errors are due to random Poisson noise. A typical error of metallicity determination using this approach for spectra of GCs with $S / N \geq 30$ is less than 0.2 dex (SAP06). Relative errors of age determination are $\sigma_{t}=\delta(\log (\mathrm{age} / \mathrm{yr})) \sim 0.2$. The results of evolutionary parameter determination using this method are presented in Cols. 2-4 of Table 5, and are illustrated in Appendix B as $\chi^{2}$ and confidence 
contours in the age $-[\mathrm{Z} / \mathrm{H}],[\alpha / \mathrm{Fe}]-[\mathrm{Z} / \mathrm{H}]$, and age $-[\mathrm{Z} / \mathrm{H}]$ planes. A description of the program is also given.

We derive low metallicities $([\mathrm{Fe} / \mathrm{H}] \leq-1.1 \mathrm{dex})$, and old ages (8-10 Gyr) for GC5, GC7, and Hodge II in good agreement with the evolutionary parameters for other GCs in NGC 147 (Hodge I, III) reported by SAP06. A spectrum of Hodge II of low signal-to-noise ratio $(S / N=14)$ was obtained by SAP0 $6^{4}$. They obtained different values for the metallicity $([\mathrm{Z} / \mathrm{H}]=-1.5 \mathrm{dex})$, and age $(10 \mathrm{Gyr})$. The $\alpha$-element ratio for Hodge II is not very accurate in this paper and in SAP06. It has a high value of $0.5 \mathrm{dex}$ with an uncertainty of 0.4 dex in both cases.

If one compares the location of the measured Lick indices with model predictions on two-dimensional index plots, it appears that some of the line strengths are not well reproduced by the models. Figure 5 shows age-metallicity, $[\alpha / \mathrm{Fe}]$-metallicity, and carbon, nitrogen, and calcium sensitive indices versus metallicity diagnostic plots (Puzia et al. 2005b). Metallicity indices insensitive to $[\alpha / \mathrm{Fe}]$ are used to construct them, namely $[\mathrm{MgFe}]^{\prime}=\{\mathrm{Mg} b \cdot(0.72 \cdot \mathrm{Fe} 5270+0.28 \cdot \mathrm{Fe} 5335)\}^{1 / 2}$, and $\langle\mathrm{Fe}\rangle=(\mathrm{Fe} 5270+\mathrm{Fe} 5335) / 2$. The Balmer line indices $(\mathrm{H} \beta$, $\left.\mathrm{H} \gamma_{A}, \mathrm{H} \delta_{A}\right)$ are sensitive to the temperature of hot main-sequence turn-off stars, metal lines arise from the coolest red-giant branch and lower main-sequence stars, and the $\mathrm{Mg}_{2}$ index is very sensitive to the $\alpha$-element abundance ratio (Worthey 1994; Worthey \& Ottaviani 1997). This is why the $\mathrm{H} \beta-[\mathrm{MgFe}]^{\prime}, \mathrm{H} \gamma-[\mathrm{MgFe}]^{\prime}$, $\mathrm{H} \beta-[\mathrm{MgFe}]^{\prime}$, and $\langle\mathrm{Fe}\rangle-\mathrm{Mg}_{2}$ diagnostic plots allow one to define the evolutionary parameters (Puzia et al. 2005b).

$\mathrm{CN}_{2}$ is sensitive to carbon abundance, while the G4300, $\mathrm{C}_{2} 4668$ line strengths are also sensitive to $\mathrm{C}$, but not sensitive to nitrogen variations (Tripicco \& Bell 1995). The GCs show insignificant deviations from the model predictions in the $\mathrm{CN}_{2}-[\mathrm{MgFe}]^{\prime}$ diagram. However, they show high G4300, $\mathrm{C}_{2} 4668$ line strengths. This may indicate that the GCs are overabundant in $\mathrm{C}$ and underabundant in $\mathrm{N}$. This qualitative result needs confirmation with higher resolution spectroscopic observations.

Figure 5 shows that the Balmer-line indices fall below the oldest model sequences, which may indicate ages older than the age of the Universe. For some GCs, the age derived from one diagnostic diagram differs from that derived from the other diagrams. The reason lies not only in random and systematical errors in measuring Lick indices, but also in probable SSP model problems.

The latter are ultimately tied to different physical conditions in dwarf galaxies, where we study GC systems, and in our Galaxy, where stars are used to construct the grids. Giant and dwarf galaxies experience very different evolutionary histories, which influence their stellar mass functions and chemical composition. Stars in dwarf galaxies are metal poorer, show lower $\alpha$-element abundance ratios, higher s-process element to Fe ratios (Venn et al. 2004; Pritzl et al. 2005). Supernovae of type Ia start to contribute at lower metallicities and at earlier ages in dwarfs in comparison to their massive neighbours. Dwarf galaxies experience a slower chemical evolution with possible short periods of higher star formation rates at young ages. As a result, specific element abundances may be observed (Venn \& Hill 2008). Additionally, it is worth noting that the stellar atmospheres interact with the interstellar medium, whose low temperature in dwarf galaxies may influence the production of resonance lines $(\mathrm{CaI}, \mathrm{NaI})$ with very low ionization potentials by definition, and molecules $\left(\mathrm{CN}, \mathrm{CH}, \mathrm{C}_{2}\right)$, on which the

\footnotetext{
${ }^{4}$ Note that the identifiers of Hodge II and III were inverted by mistake in SAP06.
}

Table 4. Correction terms of the transformation to the Lick/IDS standard system: $I_{\text {Lick }}=I_{\text {measured }}+c$.

\begin{tabular}{lrccr}
\hline \hline Index & $c$ & rms error & Index range & Units \\
\hline $\mathrm{CN} 1$ & -0.060 & 0.033 & {$[-0.07-0.4]$} & $\mathrm{mag}$ \\
$\mathrm{CN} 2$ & -0.016 & 0.013 & {$[-0.05-0.4]$} & $\mathrm{mag}$ \\
$\mathrm{Ca} 4227$ & -0.06 & 0.154 & {$[0.17-2.5]$} & $\AA$ \\
$\mathrm{G} 4300$ & 0.23 & 0.219 & {$[5.2-7.0]$} & $\AA$ \\
$\mathrm{Fe} 4384$ & -0.08 & 0.267 & {$[2.3-4.0]$} & $\AA$ \\
$\mathrm{Ca} 4455$ & 0.65 & 0.299 & {$[1.0-9.1]$} & $\AA$ \\
$\mathrm{Fe} 4531$ & -0.07 & 0.609 & {$[1.0-5.2]$} & $\AA$ \\
$\mathrm{Fe} 4668$ & 0.20 & 0.773 & {$[0.2-10.4]$} & $\AA$ \\
$\mathrm{H} \beta$ & -0.30 & 0.472 & {$[0.8-1.1]$} & $\AA$ \\
$\mathrm{Fe} 5015$ & 0.30 & 0.755 & {$[1.6-7.3]$} & $\AA$ \\
$\mathrm{Mg}$ & -0.002 & 0.029 & {$[-0.01-0.21]$} & $\mathrm{mag}$ \\
$\mathrm{Mg}{ }_{2}$ & 0.020 & 0.077 & {$[0.01-0.36]$} & $\mathrm{mag}$ \\
$\mathrm{Mgb}$ & 0.10 & 0.400 & {$[0.3-3.9]$} & $\AA$ \\
$\mathrm{Fe} 5270$ & 0.22 & 0.746 & {$[0.7-4.3]$} & $\AA$ \\
$\mathrm{Fe} 5335$ & 0.40 & 0.700 & {$[0.1-3.9]$} & $\AA$ \\
$\mathrm{Fe} 5406$ & 0.20 & 0.518 & {$[0.2-2.9]$} & $\AA$ \\
$\mathrm{H} \delta_{A}$ & -0.74 & 0.841 & {$[-7.6-0.6]$} & $\AA$ \\
$\mathrm{H} \gamma_{A}$ & -0.06 & 0.026 & {$[-11.6--3.6]$} & $\AA$ \\
$\mathrm{H} \delta_{F}$ & -0.30 & 0.076 & {$[-2.1-1.1]$} & $\AA$ \\
$\mathrm{H} \gamma_{F}$ & -0.07 & 0.154 & {$[-3.5--0.7]$} & $\AA$ \\
\hline
\end{tabular}

corresponding Lick indices, $\mathrm{Ca} 4227, \mathrm{NaD}$, and $\mathrm{CN}_{1}, \mathrm{CN}_{2}$, $\mathrm{G} 4300, \mathrm{C}_{2} 4668$, are centered. These absorption features are weakened much more than others (e.g. MgI, FeI) with the temperature growth.

\subsubsection{STECKMAP results}

We used STECKMAP (STEllar Content and Kinematics via Maximum a Posteriori) (Ocvirk et al. 2006a,b) as an independent method for determining evolutionary parameters. This nonparametric method is intended to recover kinematic properties and the stellar content of galaxies from their integrated-light spectra. It has also been applied to Galactic GCs (Koleva et al. 2008). It allows one to determine ages and metallicities of stellar systems from the corresponding parameter distributions by summing luminosity-weighted age and $[\mathrm{Fe} / \mathrm{H}]$ fractions for each SSP model bin:

Age $=\sum_{i=1}^{n} X_{i} t_{i} ; \quad[\mathrm{Fe} / \mathrm{H}]=10 \sum_{i=1}^{n} X_{i} \log \left([\mathrm{Fe} / \mathrm{H}]_{i}\right)$,

where $t_{i}$ and $[\mathrm{Fe} / \mathrm{H}]_{i}$ are the age and the metallicity of the $i$ th SSP of the basis, and $X_{i}$ is the weight (i.e. flux fraction) of this component.

The results are presented in Table 5 (Cols. 5, 6). Normalized spectra of three brightest GCs fitted using the STECKMAP program are shown in Fig. 4.

\subsubsection{Continuum shape fitting}

We were not aiming at an absolute flux calibration in our spectra, because the methods of analysis we use do not require it. A critical condition for using the above two methods is a high signal-to-noise ratio in the spectra $(S / N \geq 30)$. Atmospheric transparency was slightly variable during our observations. So 

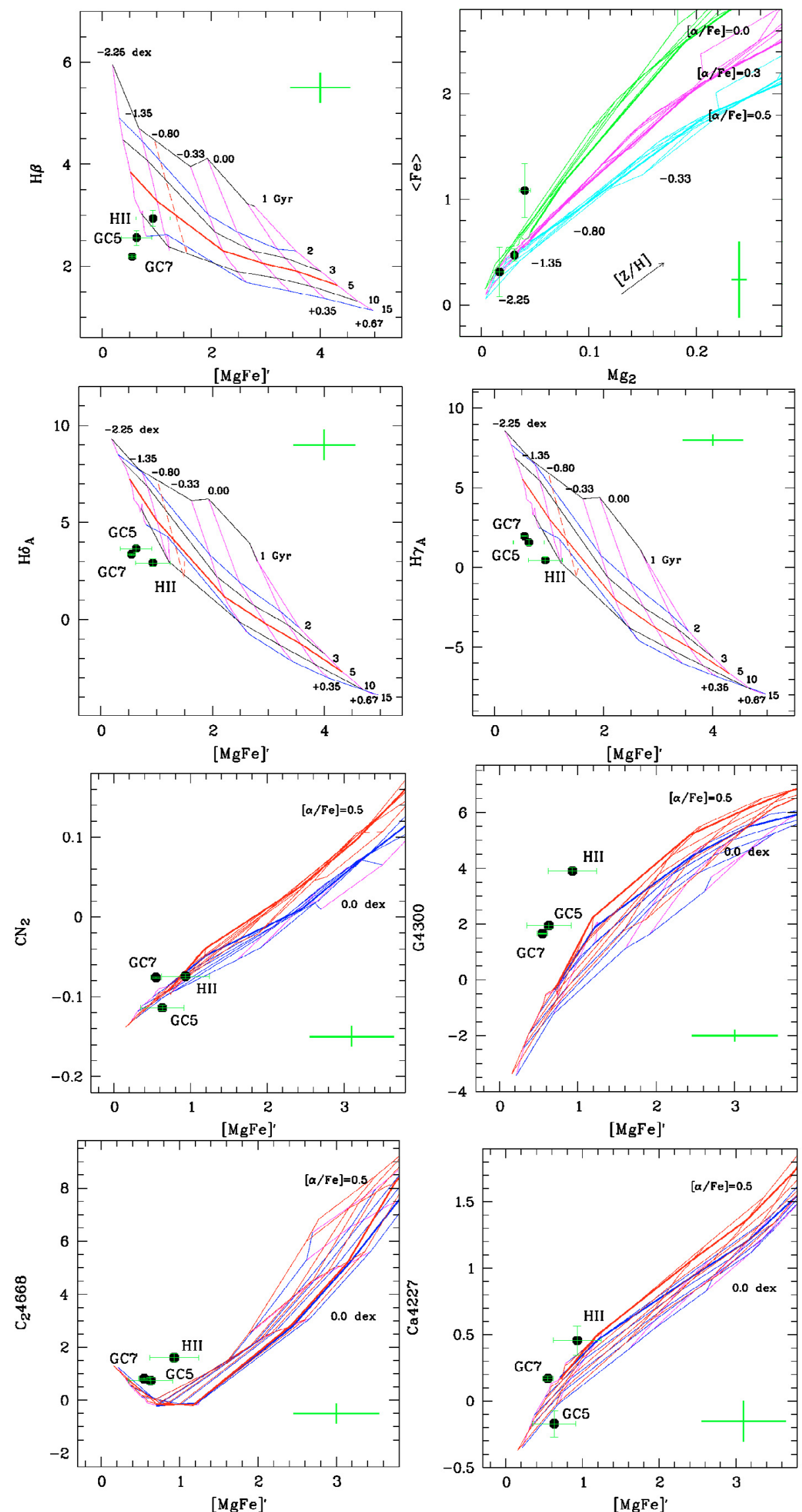

Fig. 5. Diagnostic plots of age-metallicity, $[\alpha / \mathrm{Fe}]$-metallicity, and $[\mathrm{MgFe}]^{\prime}$ versus indices sensitive to $\mathrm{C}, \mathrm{N}$, and Ca. The bootstrap errors are overplotted. We use the SSP model predictions from Thomas et al. $(2003,2004)$. Error bars on each plot show rms errors of the transformations to the Lick system. 
Table 5. Ages, metallicities and $[\alpha / \mathrm{Fe}]$ for GCs with high $S / N$ ratio obtained using three different methods (see Sect. 3.1 for details).

\begin{tabular}{lccc|cc|cr}
\hline \hline Object & $\begin{array}{c}\mathrm{Age}_{\chi^{2}} \\
\mathrm{Gyr}\end{array}$ & $\begin{array}{c}{[\mathrm{Z} / \mathrm{H}]_{\chi^{2}}} \\
\mathrm{dex}\end{array}$ & $\begin{array}{c}{[\alpha / \mathrm{Fe}]_{\chi^{2}}} \\
\text { dex }\end{array}$ & $\begin{array}{c}\mathrm{Age}_{\text {STECK }} \\
\mathrm{Gyr}\end{array}$ & $\begin{array}{c}{[\mathrm{Fe} / \mathrm{H}]_{\text {STECK }}} \\
\text { dex }\end{array}$ & $\begin{array}{c}\mathrm{Age}_{\mathrm{CS}} \\
\mathrm{Gyr}\end{array}$ & $\begin{array}{r}{[\mathrm{Fe} / \mathrm{H}]_{\mathrm{CS}}} \\
\text { dex }\end{array}$ \\
\hline GC7 & $8 \pm 2$ & $-1.5 \pm 0.2$ & $0.2 \pm 0.2$ & 8 & -1.3 & $6 \pm 2$ & $-1.6 \pm 0.2$ \\
GC5 & $10 \pm 2$ & $-1.7 \pm 0.2$ & $0.1 \pm 0.2$ & 10 & -1.5 & $10 \pm 3$ & $-1.7 \pm 0.3$ \\
Hodge II & $9 \pm 3$ & $-1.1 \pm 0.3$ & $0.5 \pm 0.4$ & 7 & -1.2 & $10 \pm 4$ & $-1.2 \pm 0.4$ \\
\hline
\end{tabular}

we decided to make use of a possible imperfection in the absolute flux calibration as an additional way of determining the evolutionary parameters.

In the following we compare our spectra of GCs with those of the PEGASE.HR models and check whether the continuum slopes and intensities of different absorption lines in our spectra and the model ones are correlated, and how they are influenced by age and metallicity. Note that, as when using the STECKMAP program, we take into account the whole spectra with all the information they contain. Our task is to find two quantities for each observed spectrum and each model age and metallicity: the slope of a multiplicative term to be applied to a GC spectrum to bring it into correspondence with a model one, and the difference between the model and the transformed object spectra. For this we first scale model and object spectra at $\lambda=5000 \AA$ and normalize simultaneously. The ratio of the normalization terms defines the miltiplicative continuum: $Z_{N}=\frac{\text { modelCONT }_{N}}{\text { objectCONT }_{\mathrm{N}}}, \mathrm{OBJ}_{N}=\operatorname{obsOBJ}_{N} \cdot Z_{N}$, where $N$ is a spectral element. Then we find the difference between the object spectra scaled at $\lambda=5000 \AA$ and multiplied by $Z_{N}$ and the corresponding model ones. Figure 6 shows the dependence between two parameters, the slope of $Z_{N}$ in its blue part $(\lambda<4700 \AA)$ and $\sigma\left(\mathrm{OBJ}_{N}-\mathrm{MODEL}_{N}\right)$ for two sets of the PEGASE-HR model spectra: 1) $Z=0.004$, ages from 1 to $14 \mathrm{Gyr}$; and 2) $Z=0.0004$, ages from 1 to 14 Gyr. The minimum standard deviation of the difference between the resulting object and model spectra at the minimum slope indicates the age and metallicity characterizing our object.

Linear interpolation between the SSP model grids and consideration of $\chi^{2}=\sum_{N} \frac{\left(\mathrm{OBJ}_{N}-\mathrm{MODEL}_{N}\right)^{2}}{\sigma\left(\mathrm{OBJ}_{N}\right)^{2}}$ parameter distribution with the observed spectrum of errors, $\sigma\left(\mathrm{OBJ}_{N}\right)$, allow one to estimate errors of age and metallicity determination. This approach is a simplification of methods used for modeling the spectral evolution of galaxies (Chilingarian 2007; Koleva et al. 2008).

The results of our spectrum fitting are presented in Table 5 (Cols. 7, 8).

\subsection{Specific frequency of GCs in NGC 147}

The number of globular clusters per unit $M_{V}=-15$ mag of host galaxy light, $S_{N}=N_{\mathrm{GC}} \times 10^{0.4\left(M_{V}+15\right)}$ (Harris \& van den Bergh 1981), is equal to 6.4 for NGC 147. This value is higher than the one expected if a constant number of GCs per unit mass are formed with a constant efficiency: $S_{N} \sim 0.0025 L_{\text {gal }}^{0.3}$ (McLaughlin 1999).

The corresponding number of GCs per unit galaxy mass $M_{\mathrm{G}}=10^{9} M_{\odot}$ is

$T=\frac{N_{\mathrm{GC}}(\text { tot })}{M_{\mathrm{G}} / 10^{9} M_{\odot}}$.

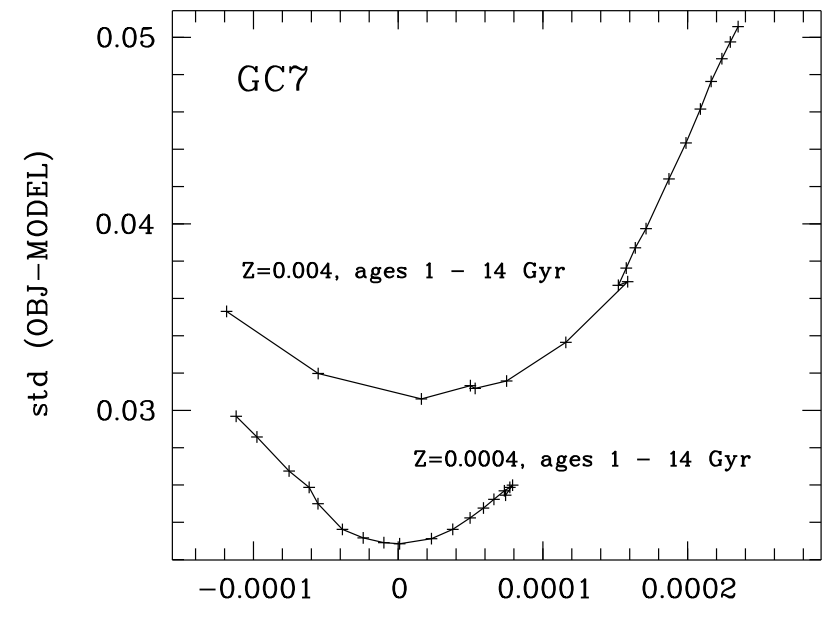

Slope of a multiplicative continuum $(\lambda<4700 \AA)$

Fig. 6. Result of division of the blue continuum slopes $(\lambda<4700 \AA)$ in the spectra of GC7 and two sets of the PEGASE-HR models of metallicities $Z=0.004$, and $Z=0.0004$ and different ages $(1-14 \mathrm{Gyr})$ versus the difference between the object and model spectra, both normalized and scaled to the flux at $\lambda<5000 \AA$.

(Zepf \& Ashman 1993) Using the mass estimate $5 \times 10^{8} M_{\odot}$ (see Appendix A), $T=14$ for NGC 147. This value is higher than for NGC $205(T=4)$, and NGC $185(T=12.5)$ using the mass estimates of Bender et al. (1991). However, it is much lower than for dark matter dominated dSphs. For example, the extremely low surface brightness dSph KK221 in the Centaurus A group has a mass $M \sim 6 \times 10^{7} M_{\odot}$ and a number of spectroscopically confirmed GCs $N_{\mathrm{GC}}=6$ (Sharina \& Puzia 2008), thus the mass fraction of GCs in that galaxy is $T=100$.

\section{Discussion}

In order to patch together a reasonable scenario for the formation history of NGC 147, we now compare the physical parameters of its GCs with those of the field stars, as well as with those of stars and GCs in other galaxies of the M 31 group.

\subsection{NGC 147}

\subsubsection{Metallicities and abundance ratios}

The evolutionary status of diffuse stellar populations in NGC 147 has been studied by many authors starting with Mould et al. (1983), who estimated a mean metallicity of 
$[\mathrm{Z} / \mathrm{H}]=-1.2 \pm 0.2 \mathrm{dex}$, with a spread of $0.3 \mathrm{dex}$, for the red giants in the outer parts of the galaxy. This coincides with the value in the stellar halo of M 31 at galactocentric distances greater than $60 \mathrm{kpc}$ (Kalirai et al. 2006). In the center of NGC 147, the metallicity is higher: $[\mathrm{Fe} / \mathrm{H}]=-0.9 \mathrm{dex}$ (Grebel 2000; McConnachie et al. 2005; Dolphin 2005). The mean abundance of planetary nebulae, $[\mathrm{Fe} / \mathrm{H}] \sim-0.97$ (Gonçalves et al. 2007), is close to the one of the old stellar populations.

By comparison, the bright and massive GCs of NGC 147 have a very low mean metal content, much lower than the halo stars (Da Costa \& Mould 1988; SAP06; Table 5 in this paper). The faint GCs Hodge I and Hodge II near the center of the galaxy show enhanced metal abundances, close to the metallicity of the oldest stars $([\mathrm{Z} / \mathrm{H}] \sim-1.0 \div-1.2 \mathrm{dex})$. All the GCs have solar $\alpha$-element abundance ratios within the errors.

\subsubsection{Ages}

NGC 147 contains a small amount of intermediate-age C and M stars (Nowotny et al. 2003; Davidge 1994, 2005). The most recent star-forming event in NGC 147 happened 3 Gyr ago, according to broad-band near-infrared color-magnitude diagrams (Davidge 2005). On the other hand, the absence of mainsequence turn-off stars with $M_{V}<-1$ indicates that the most recent large-scale star-forming activity occurred at least $1 \mathrm{Gyr}$ ago (Han et al. 1997). It may have coincided with the formation of the brightest M giants (Sohn et al. 2006). These estimates of the most recent star-forming event agree with the study of Dolphin (2005) using HST images. It is important that the brightest AGB stars in NGC 147 are well-mixed with fainter stars. This may indicate that the whole galaxy was involved in the star formation process, or that it has been tidally stirred by interactions with another galaxy.

The GCs in NGC 147 appear to be much older than the brightest AGB stars (see Table 7 in SAP06 and Table 5 in this paper). Their mean age is $9 \pm 1$ Gyr. It should be noticed, however, that the brightest clusters GC7 and Hodge III, despite their low metallicities, show rather young ages, similar to those of the more metal-rich clusters Hodge I, and II. This may be the result of artificial filling-in of the Balmer lines, caused by hot stars, such as horizontal branch (HB) and emission-line stars (Schiavon et al. 2005; Recio-Blanco et al. 2006). These artificially younger ages may also result from the presence of multiple stellar populations, since Balmer lines are mostly sensitive to the main-sequence turn-off temperature (Worthey 1994). In our Galaxy, the most massive GCs also show indications of unusually hot, extended HBs, multiple stellar populations, and are kinematically distinct from other Galactic GCs (Recio-Blanco et al. 2006; Lee et al. 2007).

\subsection{31 and other $d E$ satellites}

The absence of young or even intermediate-age GCs in NGC 147 contrasts with the situation in other dEs of the M 31 group. NGC 185 and 205 contain GCs of intermediate age and metallicity (SAP06): FJJVII ([Z/H] $=-0.8$, age $=7 \mathrm{Gyr})$ in NGC 185, Hubble V $([\mathrm{Z} / \mathrm{H}]=-0.6$, age $=1 \mathrm{Gyr})$, and Hubble VI $([\mathrm{Z} / \mathrm{H}]=$ -0.8 , age $=4$ Gyr) in NGC 205 . Such younger objects also exist in the M 31 halo (see Sect. 4.2 in SAP06 and references therein). Fan et al. (2006) found young and intermediate-age GCs in M 31, peaking at $\sim 3$ and 8 Gyr from multicolor photometry.
Spectroscopic studies have shown that three types of GC populations exist in M 31: old objects with a wide range of metallicities, intermediate age and metallicity GCs, and young GCs with slightly higher metallicity (Perrett et al. 2002; Beasley et al. 2004, 2005; Fusi Pecci et al. 2005; Puzia et al. 2005a).

Stellar populations of intermediate ages and metallicities are also present in the close neighbourhood of M 31. Mean metallicities of stars and metallicity spreads are greater in NGC 205, and 185 , respectively $-0.5 \pm 0.5$, and $-0.8 \pm 0.4$ (Grebel 2000). The spectroscopic age of M 32 is 2-3.5 Gyr (Schiavon et al. 2004). Brown et al. (2003) estimated that $\sim 30 \%$ of the halo old population in M 31 comprises intermediate-age stars with ages of 6-8 Gyr, and metallicities of $\sim-0.5 \mathrm{dex}$.

The spectroscopically studied M 31 GCs are poorer in $\alpha$-elements than Galactic GCs: $[\alpha / \mathrm{Fe}]=0.14 \pm 0.04$ dex (Pritzl et al. 2005; Puzia et al. 2005a), and GCs in the M 31 group dEs have near-solar $\alpha$-element abundance ratios (SAP06).

\subsection{How did NGC 147 evolve?}

All the aforementioned literature data about ages and metallicities of stellar populations indicate that there were two main star forming events in the neighbourhood of M 31: 1 $\div 3$ and $\sim 8 \mathrm{Gyr}$ ago. In spite of the large errors associated with the evolutionary parameters, the ages of GCs in NGC 147, 185 and 205 concentrate around these two periods. Detailed theoretical and observational studies will help to identify the cause of this bimodality. It might be the tidal disruption of a satellite galaxy, or interactions between M 31 and M 33.

The globular cluster system of NGC 147 formed during early and very powerful star forming events in the life of the galaxy (Da Costa \& Mould 1988). From the age spread of the GCs, this active period started $\sim 8 \div 10 \mathrm{Gyr}$ ago, and continued for $\sim 2$ Gyr. The massive clusters Hodge III, GC5 and GC7 have lower metallicities than the mean metallicity of metal-poor stars in the outer parts of NGC 147 and in the M 31 halo. This is an indication of very early formation. The faint clusters Hodge I and Hodge II and the majority of the halo stars formed later and simultaneously, since their metallicities are similar. The difference in metallicities between the youngest low-mass GCs and the youngest stars is only $\sim 0.3 \mathrm{dex}$. It is thus likely that the gas content was already low after the first starburst, and the metallicity has not changed much since. Finally, after a weaker period of star formation approximately 3 Gyr ago (Davidge 2005), the galaxy was depleted of the rest of its gas.

It is interesting that all the outer GCs are located west and south-west of the galaxy center. This may result from tidal interaction or ram-pressure stripping due to motion through dense gas, or else from anisotropic galactic-scale winds, which would also explain the gas depletion in the galaxy.

\section{Conclusion}

We have reported on the discovery and spectroscopic study of three new GCs in NGC 147. Medium-resolution spectra were obtained at the 6-m telescope, and radial velocities were measured for all the objects. The clusters GC5, GC7, and GC10 have heliocentric radial velocities close to that of NGC 147, while other candidates appear to be remote galaxies. The newly found GCs are anisotropically distributed: they are located at projected distances $0.9-2.1 \mathrm{kpc}$ south-west of the center of the galaxy. 
We obtained evolutionary parameters for the brightest globular clusters GC5 and GC7, and for Hodge II. They appear to be old and metal-poor. Although the evolutionary parameters, derived using medium-resolution spectra, have large uncertainties, the ages and metallicities of GCs in NGC 147 show clear correlations with those of red giant branch stars in the galaxy and of GCs and old stars in the M 31 halo. Thus the bright clusters Hodge III, GC5, and GC7 were likely to be formed first. Their metallicities are lower than the mean metallicity of the old stars in the galaxy: $[\mathrm{Z} / \mathrm{H}] \sim-1.5 \div-1.8$. The fainter clusters Hodge I and Hodge II are second generation objects. They show metallicities similar to that of the oldest stars in the galaxy, $[\mathrm{Z} / \mathrm{H}]=-1.2$. We suggest that the main star forming period occurred $\sim 8-10$ Gyr ago. Then the activity was slow, and the rest of the gas was depleted $\sim 1 \div 3 \mathrm{Gyr}$ ago.

Hodge III, and GC7, the brightest GCs in NGC 147, seem rather young, given their low metallicities. We explain this by the possible presence of multiple stellar populations and unusually hot HB stars.

The mass of NGC 147 from the projected positions and radial velocities of the GCs is $\sim 5 \times 10^{8} M_{\odot}$, in good agreement with previous estimates. The updated specific frequency and mass fraction of GCs in NGC 147 are higher than those of NGC 185 and NGC 205.

Deep color-magnitude diagrams and high-resolution spectra of GCs obtained with telescopes in space will be needed to further progress in our understanding of the evolutionary history of NGC 147.

Acknowledgements. MES gratefully acknowledges financial support from the Centre National de la Recherche Scientifique (France), Russian Foundation for Basic Research grants 08-02-00627, and 08-02-08-536 3 , and deeply thanks Observatoire Midi-Pyrénées for its hospitality. We thank Gérard Lelièvre for help with the CFHT observations, and J. F. Le Campion for determining accurate positions for our GCCs; Dmitrij Makarov, Gretchen Harris, Thomas Puzia, Slava Shimansky and an anonymous referee for valuable comments; Pierre Ocvirk and Thomas Puzia for help in using of their programs STECKMAP and GONZO. Preliminary photometric and structural investigations of the data were done by Thierry Devilière. We are grateful to the Large Telescopes Program Committee of the Russian Academy of Sciences for allocation of observing time.

\section{Appendix A: Mass estimate for NGC 147}

In the following we estimate the mass of NGC 147 from the projected position and line-of-sight velocity of GCs obtained in this paper and by SAP06. We use the "tracer mass estimator" (Evans 2003), applied when the tracer population does not follow the dark matter density profile. In the case of an isothermal potential:

$M_{\text {press }}=\frac{C}{G N} \sum_{i}\left(v_{i, \text { los }}-\langle v\rangle\right)^{2} R_{i}$,

where

$C=\frac{16(\gamma-2 \beta)}{\pi(4-3 \beta)} \cdot \frac{4-\gamma}{3-\gamma} \cdot \frac{1-\left(r_{\text {in }} / r_{\text {out }}\right)^{3-\gamma}}{1-\left(r_{\text {in }} / r_{\text {out }}\right)^{4-\gamma}}$.

Here, $\langle v\rangle$ is the system's mean radial velocity and $\beta$ the anisotropy parameter $1-\sigma_{t}^{2} / \sigma_{r}^{2}$, equal unity for purely radial orbits and $-\infty$ for a system with solely tangential orbits (Binney 1981).

We used the power-law rule $\gamma=1+\mathrm{d} \log \Sigma / \mathrm{d} \log R$ (Gebhardt et al. 1996) to derive the three-dimensional density profile of the GC population. If the surface-brightness profile is purely exponential, then $\gamma=2$ (De Rijcke et al. 2006). We estimate the Sersic indices $n$ along the major and minor axes the galaxy to be $n=0.86$, and $n=1.0$, respectively, using the surface-brightness profiles (Kent 1987). These values are in a good agreement with the conclusion of De Rijcke et al. (2006).

There are different estimates of the rotation velocity for NGC 147 in the literature. According to De Rijcke et al. (2006), it is equal to zero. Bender et al. (1991) found rotation with an amplitude $V_{\mathrm{r}} \sim 10 \mathrm{~km} \mathrm{~s}^{-1}$.

The mass of NGC 147 is equal to $M_{\text {press }} \approx 6.2 \pm 2 \times 10^{8} M_{\odot}$ from Eqs. (A.1) and (A.2) in the case of $V_{\mathrm{r}} \sim 0 \mathrm{~km} \mathrm{~s}^{-1}$, the anisotropy parameter $\beta=0.5$ for randomly oriented orbits, and radii of orbits of the nearest and most distant GC: $r_{\text {in }}=1.2 \mathrm{pc}$ and $r_{\text {out }}=2.08 \mathrm{kpc}$. The statistically unbiased estimate of the mass value is $M_{\text {press }}^{c}=M_{\text {press }}\left[1-\left(2 \sigma_{v}^{2}\right) / 3 s_{v}^{2}\right] \sim M_{\text {press }} \cdot 0.8 \sim$ $5 \times 10^{8} M_{\odot}$, where $\sigma_{v}$ is the rms error of the radial velocity measurements, and $s_{v}$ is the rms velocity of GCs relative to the mean velocity of the GC system, $s_{v}^{2}=(1 / k) \sum\left(v_{k}-\langle v\rangle\right)^{2}$ with a number of GCs equal $k$ (Karachentsev et al. 1999).

The expected uncertainty of the total mass estimate is $\sim 50 \%$ taking into account the small number of GCs. In the present case of seven GCs, the error on the velocity dispersion measurement is $\sim 23 \%$ of the value of the velocity dispersion itself. In spite of this large uncertainty the obtained mass and $M / L_{B}$ estimate are in good agreement with those of Bender et al. (1991).

\section{Appendix B: Comparison of our Lick index measurements with SSP model predictions using a 3-dimensional interpolation and $\chi^{2}$ minimization routine}

In this section we illustrate the results described in Sect. 3.1.1.

With our method (see also SAP06), we first obtain a full set of theoretical Lick indices for any age, metallicity and $[\alpha / \mathrm{Fe}]$ via three-dimensional linear interpolation. Then we minimize the following $\chi^{2}$ function:

$\chi^{2}=\sum_{i=1}^{N}\left(\frac{I_{i}-I_{i}(\text { age },[\mathrm{Z} / \mathrm{H}],[\alpha / \mathrm{Fe}])}{\sigma_{I_{i}}}\right)^{2}$,

where $N$ is the number of Lick indices involved in the analysis, $I_{i}$ is an observed index, $\sigma_{I_{i}}$ is the total uncertainty of the index, including rms error of transformations to the Lick/IDS system, $I_{i}($ age $,[\mathrm{Z} / \mathrm{H}],[\alpha / \mathrm{Fe}])$ is the theoretical index prediction.

A contour plot in Figs. B.1-B.6 shows isolines of threedimensional $\chi^{2}$ solution space, sliced at the point of the global minimum. The contour $\chi^{2}$ levels and the corresponding $67 \%$ 95\% and $99 \%$ confidence plots are shown. See Sect. 3.1.1 for a discussion about the advantages and limitations of using of Lick indices and the $\chi^{2}$ minimization technique for evolutionary parameters determination. 
fixed $[\alpha / \mathrm{Fe}]$

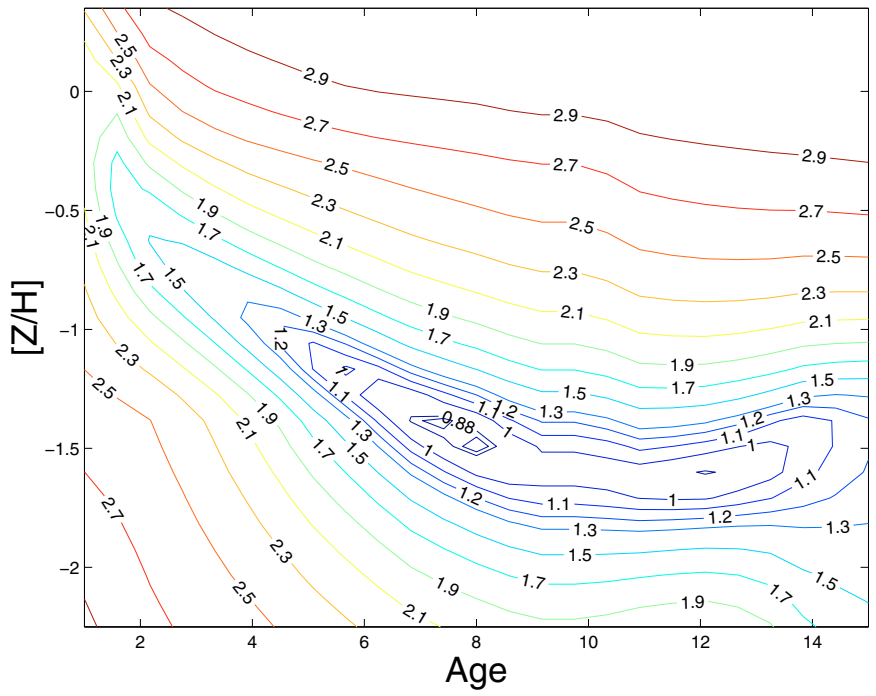

fixed Age

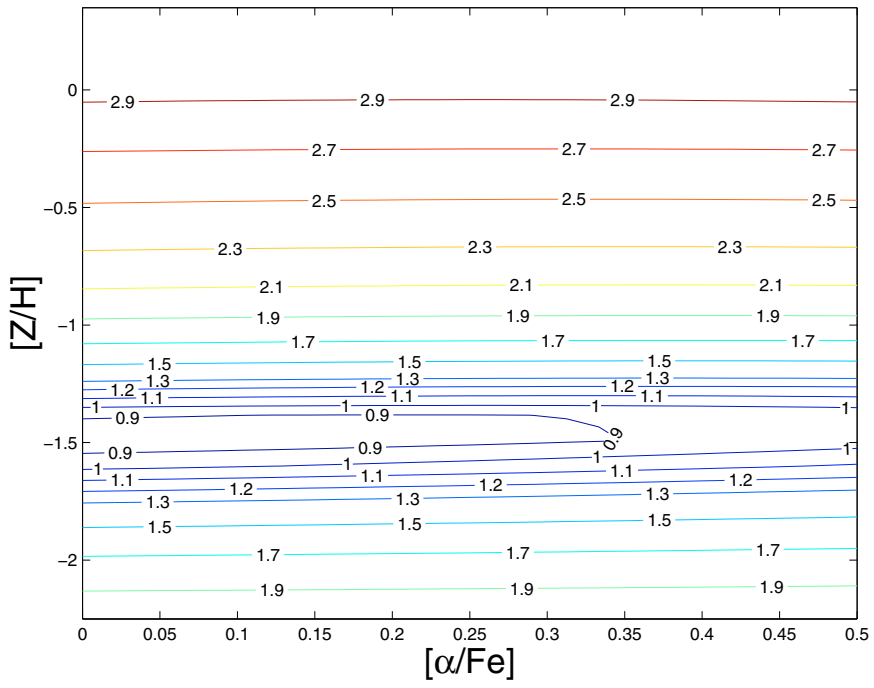

fixed $[\mathrm{Z} / \mathrm{H}]$

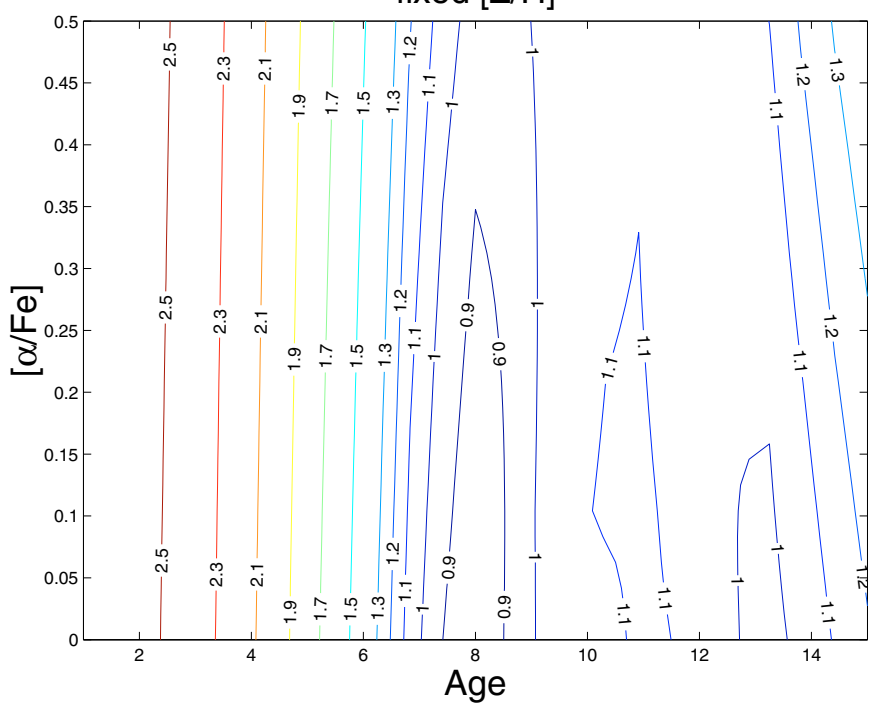

Fig. B.1. GC7: Age- $[\mathrm{Z} / \mathrm{H}],[\alpha / \mathrm{Fe}]-[\mathrm{Z} / \mathrm{H}]$, and Age- $[\alpha / \mathrm{Fe}]$ slices of the three-dimensional $\chi^{2}$ space taken at the point of the global minimum. fixed $[\alpha / \mathrm{Fe}]$
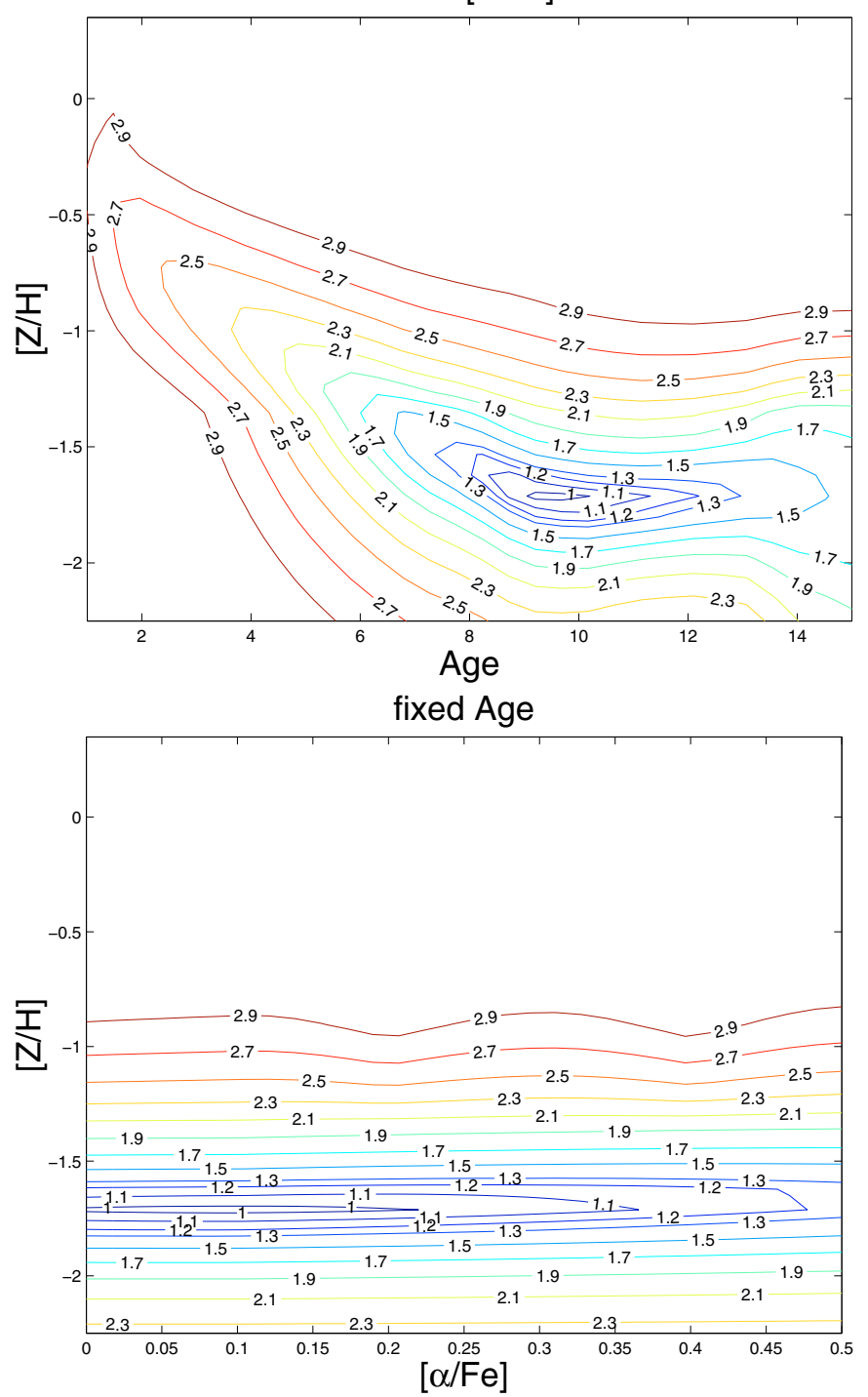

fixed $[\mathrm{Z} / \mathrm{H}]$

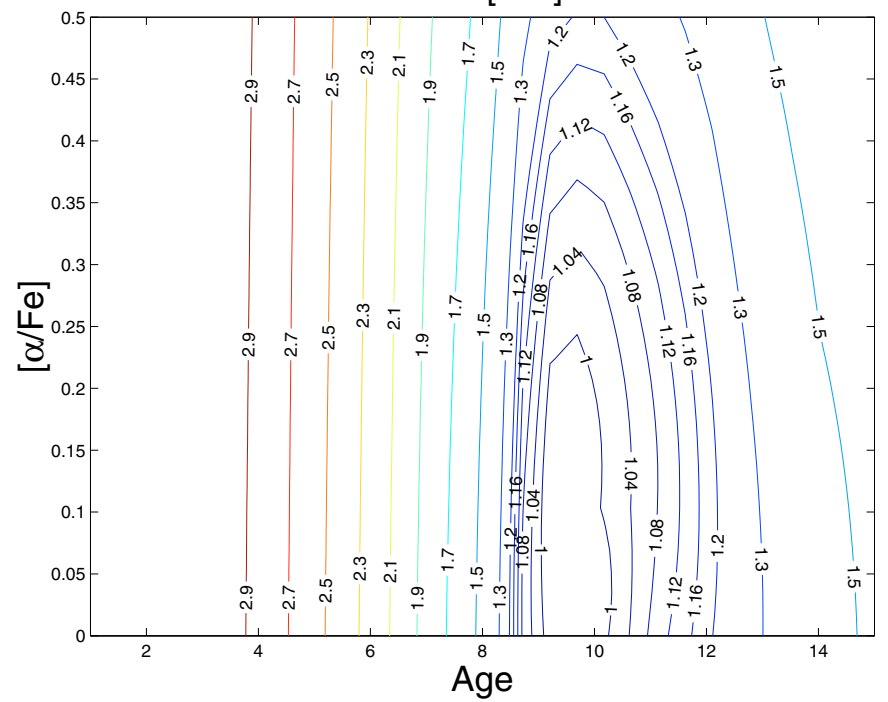

Fig. B.2. Same as in Fig. B.1, but for GC5. 
fixed $[\alpha / F e]$
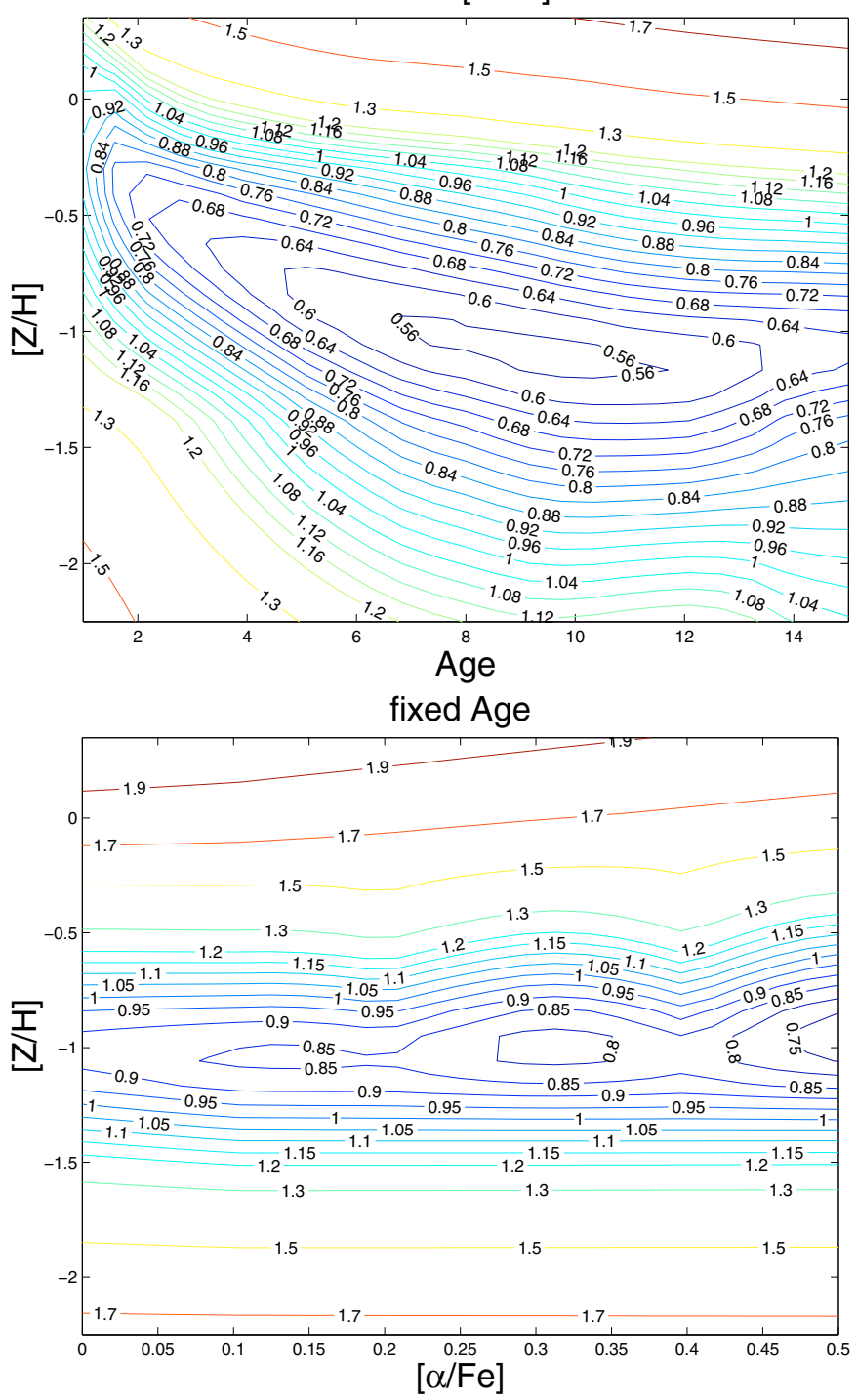

fixed $[\mathrm{Z} / \mathrm{H}]$

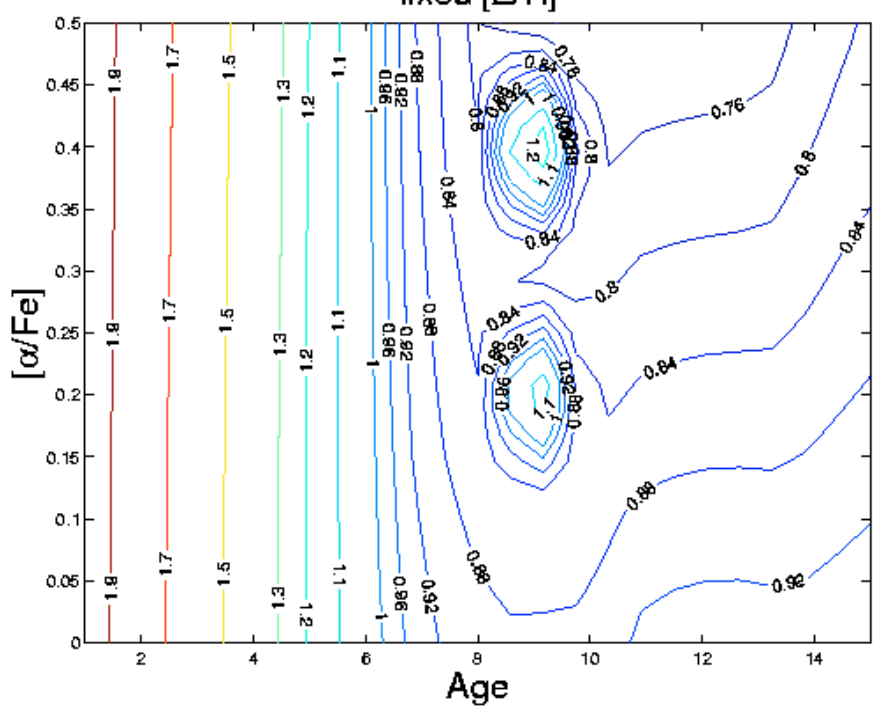

Fig. B.3. Same as in Fig. B.1, but for Hodge II. fixed $[\alpha / \mathrm{Fe}]$
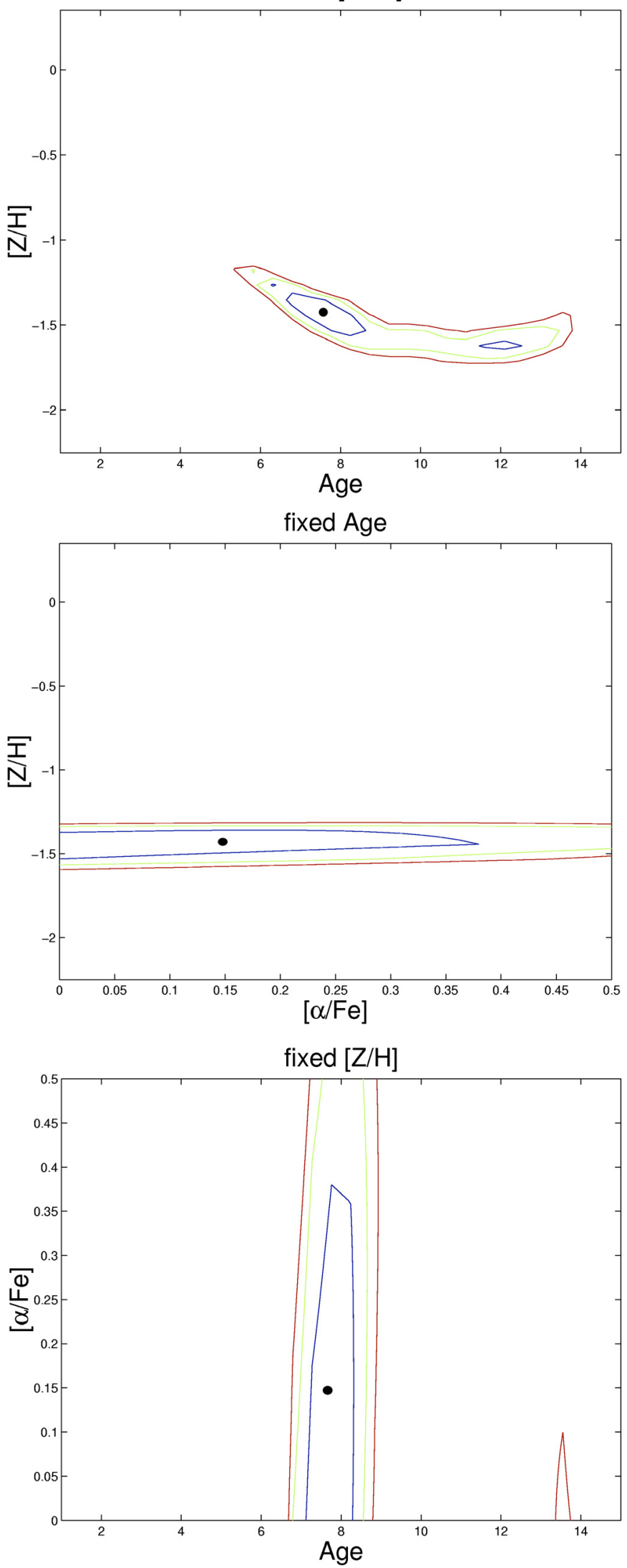

Fig. B.4. GC7: 67\% 95\% and 99\% confidence plots in the Age- $[\mathrm{Z} / \mathrm{H}]$, $[\alpha / \mathrm{Fe}]-[\mathrm{Z} / \mathrm{H}]$, and Age $-[\alpha / \mathrm{Fe}]$ planes. 

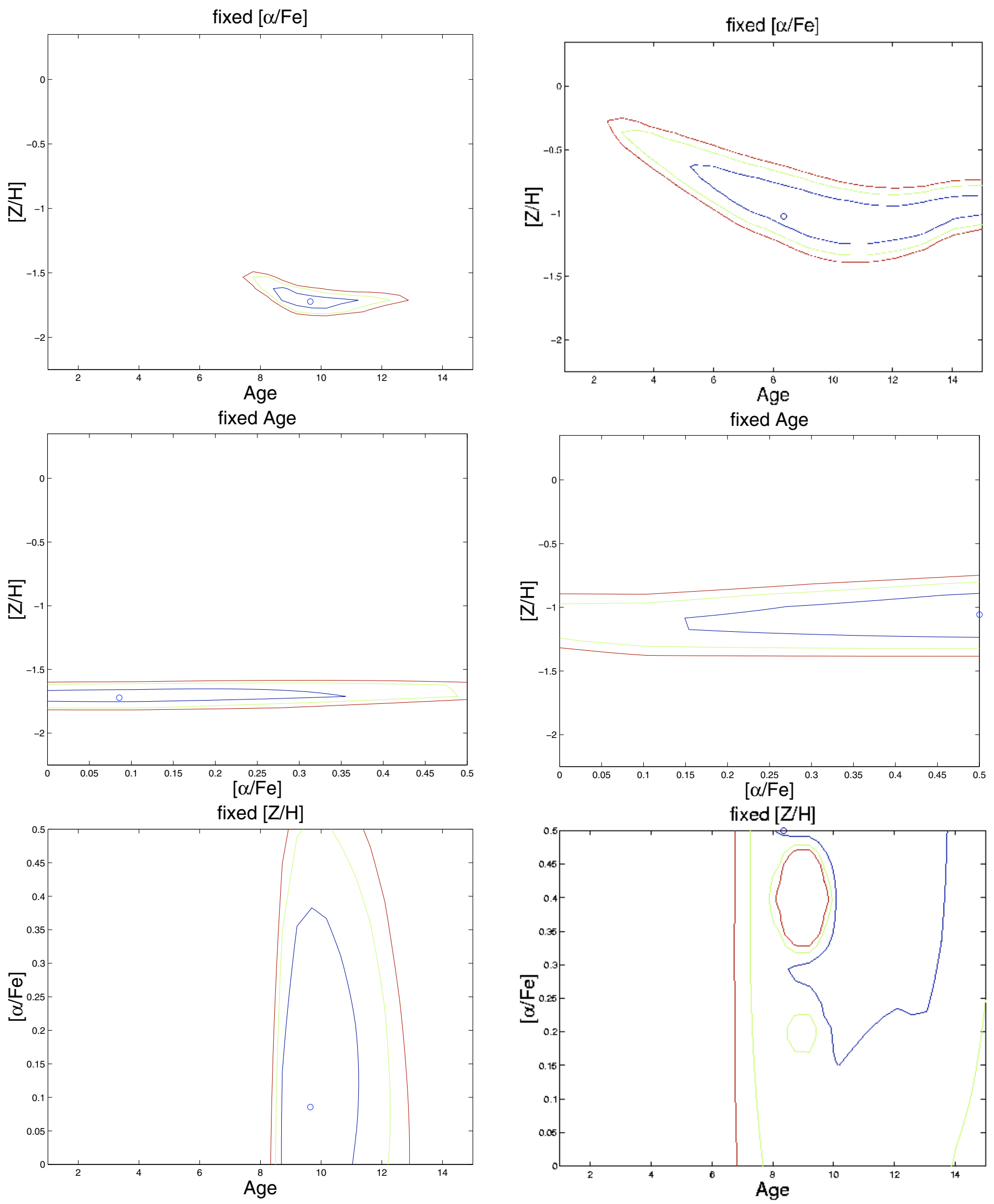

Fig. B.5. Same as in Fig. B.1, but for GC5.

Fig. B.6. Same as in Fig. B.1, but for Hodge II. 
Appendix C: Lick index measurements

Table C.1. Globular cluster indices $(\lambda \leq 4531 \AA$ ) (first line) corrected for zeropoints of transformation to the standard Lick system and errors determined from bootstrapping of the object spectrum (second line). The $S / N$ ratios per pixel in the spectra of the clusters Hodge II, GC5 and GC7, measured at $5000 \AA$ are respectively $\sim 26,30$ and 99 .

\begin{tabular}{|c|c|c|c|c|c|c|c|c|c|c|c|}
\hline ID & & $\begin{array}{l}\mathrm{H} \delta_{\mathrm{A}} \\
(\AA)\end{array}$ & $\begin{array}{r}\mathrm{H} \gamma_{\mathrm{A}} \\
(\AA)\end{array}$ & $\begin{array}{r}\mathrm{H} \delta_{\mathrm{F}} \\
(\AA)\end{array}$ & $\begin{array}{r}\mathrm{H} \gamma_{\mathrm{F}} \\
(\AA)\end{array}$ & $\begin{array}{r}\mathrm{CN}_{1} \\
(\mathrm{mag})\end{array}$ & $\begin{array}{l}\mathrm{CN}_{2} \\
(\mathrm{mag}\end{array}$ & $\begin{array}{r}\mathrm{Ca} 4227 \\
(\AA)\end{array}$ & $\begin{array}{r}\mathrm{G} 4300 \\
(\AA)\end{array}$ & $\begin{array}{r}\mathrm{Fe} 4383 \\
(\AA)\end{array}$ & $\begin{array}{r}\mathrm{Ca} 4455 \\
(\AA)\end{array}$ \\
\hline \multirow[t]{2}{*}{ Hodge II } & & 2.92 & 0.46 & 2.48 & 0.99 & -0.118 & -0.074 & 0.46 & 3.91 & -0.51 & 0.65 \\
\hline & \pm & 0.20 & 0.20 & 0.21 & 0.21 & 0.002 & 0.003 & 0.11 & 0.11 & 0.13 & 0.13 \\
\hline \multirow[t]{2}{*}{ GC5 } & & 3.65 & 1.58 & 2.45 & 2.32 & -0.156 & -0.114 & -0.17 & 1.95 & 1.47 & 0.48 \\
\hline & \pm & 0.18 & 0.18 & 0.19 & 0.19 & 0.002 & 0.003 & 0.10 & 0.11 & 0.12 & 0.12 \\
\hline \multirow[t]{2}{*}{ GC7 } & & 3.36 & 1.98 & 2.58 & 2.24 & -0.141 & -0.076 & 0.17 & 1.66 & 0.01 & 0.56 \\
\hline & \pm & 0.04 & 0.04 & 0.04 & 0.04 & 0.001 & 0.001 & 0.02 & 0.02 & 0.03 & 0.03 \\
\hline
\end{tabular}

Table C.2. Globular cluster indices $(\lambda \geq 4531 \AA$ ) (first line) corrected for zeropoints of transformation to the standard Lick system and errors determined from bootstrapping of the object spectrum (second line).

\begin{tabular}{lllrrrrrrrrr}
\hline \hline ID & $\begin{array}{l}\text { Fe4531 } \\
(\AA)\end{array}$ & $\begin{array}{r}\mathrm{Fe} 4668 \\
(\AA)\end{array}$ & $\begin{array}{r}\mathrm{H} \beta \\
(\AA)\end{array}$ & $\begin{array}{r}\mathrm{Fe} 5015 \\
(\AA)\end{array}$ & $\begin{array}{r}\mathrm{Mg} 1 \\
(\mathrm{mag})\end{array}$ & $\begin{array}{r}\mathrm{Mg} 2 \\
(\mathrm{mag})\end{array}$ & $\begin{array}{r}\mathrm{Mgb} \\
(\AA)\end{array}$ & $\begin{array}{r}\mathrm{Fe} 5270 \\
(\AA)\end{array}$ & $\begin{array}{r}\mathrm{Fe} 5335 \\
(\AA)\end{array}$ & $\begin{array}{r}\mathrm{Fe} 5406 \\
(\AA)\end{array}$ \\
\hline Hodge II & 3.32 & 1.61 & 2.94 & 2.21 & 0.031 & 0.040 & 0.67 & 1.56 & 0.81 & 1.48 \\
& \pm & 0.14 & 0.16 & 0.16 & 0.17 & 0.005 & 0.005 & 0.18 & 0.18 & 0.18 & 0.18 \\
$\mathrm{GC5}$ & & 0.99 & 0.75 & 2.56 & 1.57 & 0.007 & 0.017 & 1.14 & 0.70 & 0.33 & 0.51 \\
& \pm & 0.13 & 0.14 & 0.15 & 0.16 & 0.004 & 0.004 & 0.16 & 0.16 & 0.17 & 0.17 \\
GC7 & & 0.72 & 0.82 & 2.19 & 1.61 & 0.016 & 0.030 & 0.62 & 0.50 & 0.65 & 0.75 \\
& \pm & 0.03 & 0.03 & 0.03 & 0.03 & 0.001 & 0.001 & 0.04 & 0.04 & 0.04 & 0.04 \\
\hline
\end{tabular}

\section{References}

Afanasiev, V. L., \& Moiseev, A. V. 2005, Astron. Lett., 31, 194

Baade, W. 1944a, ApJ, 100, 137

Baade, W. 1944b, ApJ, 100, 147

Banse, K., Crane, Ph., Ounnas, Ch., \& Ponz, D. 1983, MIDAS, in Proc. DECUS, Zurich, 87

Battinelli, P., \& Demers, S. 2004, A\&A, 417, 479

Beasley, M. A., Brodie, J. P., Strader, J., et al. 2004, AJ, 128, 1623

Beasley, M. A., Brodie, J. P., Strader, J., et al. 2005, AJ, 129, 1412

Bekki, K., Couch, W. J., Drinkwater, M. J., \& Gregg, M. D. 2001, ApJ, 557, L39

Bender, R., Paquet, A., \& Nieto, J.-L. 1991, A\&A, 24, 349

Binggeli, B., Sandage, A., \& Tammann, G. A. 1985, AJ, 90, 1681

Binney, J. 1981, Structure and Evolution of Normal Galaxies, ed. D. Lynden-Bell, \& S. M. Fall (Cambridge: Cambridge University Press)

Brown, T. M., Ferguson, H. C., Smith, E., et al. 2003, ApJ, 592, L17

Butler, D. J., \& Martínez-Delgado, D. 2005, AJ, 129, 2217

Chilingarian, I. V., Prugniel, Ph., Sil'chenko, O. K., \& Afanasiev, V. L. 2007, MNRAS, 376, 1033

Corradi, R. L. M., \& Schwarz, H. E. 2005, A\&A, 431, 555

Da Costa, G. S., \& Mould, J. R. 1988, ApJ, 334, 159

Davidge, T. J. 1994, AJ, 108, 2123

Davidge, T. J. 2005, AJ, 130, 2087

De Rijcke, S., Prugniel, P., Simien, F., \& Dejonghe, H. 2006, MNRAS, 369, 1321

Dolphin, A. E. 2005, in Resolved Stellar Populations, ed. D. Valls-Gabaud, \& M. Chavez, ASP Conf. Ser., TBA, 74

Evans, N. W., Wilkinson, M. I., Perrett, K. M., \& Bridges, T. J. 2003, ApJ, 583, 752

Faber, S. M. 1973, ApJ, 179, 731

Fan, Z., Ma, J., de Grijs, R., Yang, Y., \& Zhou, X. 2006, MNRAS, 371, 1648

Ferguson, H. C. 1989, AJ, 98, 367

Ford, H. C., Jacoby, G., \& Jenner, D. C. 1977, ApJ, 213, 18

Fusi Pecci, F., Bellazzini, M., Buzzoni, A., De Simone, E., \& Federici, L. 2005, AJ, 130,554

Gebhardt, K., Richstone, D., Ajhar, E. A., et al. 1996, AJ, 112, 105
Gonçalves, D. R., Magrini, L., Leisy, P., \& Corradi, R. L. M. 2007, MNRAS, 375,715

Graham, A. W. 2002, ApJ, 568, L13

Grebel, E. K. 2000, in Star Formation from the Small to the Large Scale, ed. F. Favata, A. A. Kaas, \& A. Wilson (Noordwijk: ESA), 33rd ESLAB Symp., SP-445, 87

Han, M., Hoessel, J. G., Gallagher, J. S., III, Holtsman, J., \& Stetson, P. B. 1997, AJ, 113, 1001

Harris, W. E. 1991, ARA\&A, 29, 543

Harris, W. E., \& van den Bergh, S. 1981, AJ, 86, 1627

Hodge, P. W. 1963, AJ, 68, 691

Hodge, P. W. 1976, AJ, 81, 25

Horne, K. 1986, PASP, 98, 609

Jordán, A., Côté, P., Blakeslee, J. P., et al. 2005, ApJ, 634, 1002

Kalirai, J. S., Gilbert, K. M., Guhathakurta, P., et al. 2006, AJ, 648, 389

Karachentsev, I. D., Byrd, G. G., Chernin, A. D., \& Valtonen, M. J. 1999, Fundam. Cosmic Phys., 20, 383

Karachentsev, I. D., Karachentseva, V. E., Huchtmeier, W. K., \& Makarov, D. I. 2004, AJ, 127, 2031

Karachentseva, V. E., Karachentsev, I. D., \& Börngen, F. 1985, A\&AS, 60, 213

Kent, S. M. 1987, AJ, 94, 306

Kinney, A. L., Calzetti, D., Bohlin, R. C., et al. 1996, ApJ, 467, 38

Koleva, M., Prugniel, Ph., Ocvirk, P., Le Borgne, D., \& Soubiran, C. 2008, MNRAS, 385, 1998

Le Borgne, D., Rocca-Volmerange, B., Prugniel, Ph., et al. 2004, A\&A, 425, 881

Lee, M. G., Freedman, W. L., \& Madore, B. F. 1993, AJ, 106, 964

Lee, Y. W., Gim, H. B., \& Casetti-Dinescu, D. I. 2007, ApJ, 661, L49

Mateo, M. 1998, ARA\&A, 36, 435

McConnachie, A. W., Irwin, M. J., Lewis, G. F., et al. 2004, MNRAS, 351, L94

McConnachie, A. W., Irwin, M. J., Ferguson, A. M. N., et al. 2005, MNRAS, 356, 979

McLaughlin, D. E. 1999, AJ, 117, 2398

Mould, J., Kristian, J., \& Da Costa, G. S. 1983, ApJ, 270, 471

Nowotny, W., Kerschbaum, F., Olofsson, H., \& Schwarz, H. Z. 2003, A\&A, 403, 93 
Ocvirk, P., Pichon, C., Lançon, A., \& Thiébaut, E. 2006a, MNRAS, 365, 46 Ocvirk, P., Pichon, C., Lançon, A., \& Thiébaut, E. 2006b, MNRAS, 365, 74 Oke, J. 1990, AJ, 99, 1621

Perrett, K. M., Bridges, T. J., Hanes, D. A., et al. 2002, AJ, 123, 2490

Pritzl, B. J., Venn, K. A., \& Irwin, M. 2005, AJ, 130, 2140

Proctor, R. N., Forbes, D. A., \& Beasley, M. A. 2004, MNRAS, 355, 1327

Puzia, T. H., \& Sharina, M. E. 2008, ApJ, 674, 909

Puzia, T. H., Saglia, R. P., Kissler-Patig, M., et al. 2002, A\&A, 395, 45

Puzia, T. H., Perrett, K. M., \& Bridges, T. J. 2005a, A\&A, 434, 909

Puzia, T. H., Kissler-Patig, M., Thomas, D., et al. 2005b, A\&A, 439, 997

Recio-Blanco, A., Aparicio, A., Piotto, G., de Angeli, F., \& Djorgovski, S. G. 2006, A\&A, 452, 875

Richer, M. G., \& McCall, M. L. 1995, ApJ, 445, 642

Sage, L. J., Welch, G. A., \& Mitchell, G. F. 1998, ApJ, 507, 726

Saha, A., Hoessel, J. G., \& Mossman, A. E. 1990, AJ, 100, 108

Schiavon, R. P., Caldwell, N., \& Rose, J. A. 2004, 127, 1513

Schlegel, D. J., Finkbeiner, D. P., \& Davis, M. 1998, ApJ, 500, 52

Sharina, M. E., \& Puzia, T. H. 2008, ApJ, 674, 909

Sharina, M. E., \& Puzia, T. H. 2009, in prep.

Sharina, M. E., Afanasiev, V. L., \& Puzia, T. H. 2006a, AstL, 32, 185
Sharina, M. E., Afanasiev, V. L., \& Puzia, T. H. 2006b, MNRAS, 372, 1259 (SAP06)

Sohn, Y.-J., Kang, A., Rhee, J., et al. 2006, A\&A, 445, 69

Thomas, D., Maraston, C., \& Bender, R. 2003, MNRAS, 339, 897

Thomas, D., Maraston, C., \& Korn, A. 2004, MNRAS, 351, L19

Tonry, J., \& Davis, M. 1979, AJ, 84, 1511

Tripicco, M. J., \& Bell, R. A. 1995, AJ, 110, 3035

van den Bergh, S. 1998, AJ, 116, 1688

van den Bergh, S. 2000, The Galaxies of the Local Group (Cambridge: Cambridge Univ. Press)

Venn, K. A., \& Hill, V. M. 2008, ESO Messenger, 134 (Supplement December), 23

Venn, K. A., Irwin, M., Shetrone, M. D., et al. 2004, AJ, 128, 1177

Welch, G. A., Sage, L. J., \& Mitchell, G. F. 1998, ApJ, 499, 209

Worthey, G. 1994, ApJS, 95, 107

Worthey, G., \& Ottaviani, D. L. 1997, ApJS, 111, 377

Worthey, G., Faber, S. M., Gonzalez, J. J., \& Burstein, D. 1994, ApJS, 94, 687

Young, L. M., \& Lo, K. Y. 1997, AJ, 476, 127

Zepf, S. E., \& Ashman, K. M. 1993, MNRAS, 264, 611 